\title{
Multi-modal determination of Rayleigh dispersion and attenuation curves using the circle fit method
}

\author{
R. Verachtert *, G. Lombaert, and G. Degrande \\ Department of Civil Engineering, KU Leuven, Kasteelpark Arenberg 40, B-3001 Leuven, Belgium
}

11 April 2018

\begin{abstract}
SUMMARY
This paper introduces the circle fit method for the determination of multi-modal Rayleigh dispersion and attenuation curves as part of a Multi-Channel Analysis of Surface Waves (MASW) experiment. The wave field is transformed to the frequency-wavenumber (fk) domain using a discretized Hankel transform. In a Nyquist plot of the fk-spectrum, displaying the imaginary part against the real part, the Rayleigh wave modes correspond to circles. The experimental Rayleigh dispersion and attenuation curves are derived from the angular sweep of the central angle of these circles. The method can also be applied to the analytical fk-spectrum of the Green's function of a layered halfspace in order to compute dispersion and attenuation curves, as an alternative to solving an eigenvalue problem.

A MASW experiment is subsequently simulated for a site with a regular velocity profile and a site with a soft layer trapped between two stiffer layers. The performance of the circle fit method to determine the dispersion and attenuation curves is compared with the peak picking method and the half-power bandwidth method. The circle fit method is found to be the most accurate and robust method for the determination of the dispersion curves. When determining attenuation curves, the circle fit method and half-power bandwidth method are accurate if the mode exhibits a sharp peak in the fk-spectrum. Furthermore, simulated and theoretical attenuation curves determined with the circle fit method agree very well. A similar correspondence is not obtained when using the half-power bandwidth method.

Finally, the circle fit method is applied to measurement data obtained for a MASW experiment at a site in Heverlee, Belgium. In order to validate the soil profile obtained from the inversion procedure, force-velocity transfer functions were computed and found in good correspondence with the experimental transfer functions, especially in the frequency range between $5 \mathrm{~Hz}$ and $80 \mathrm{~Hz}$.
\end{abstract}

Key words: Joint inversion - numerical approximation and analysis - seismic attenuation surface waves and free oscillations - wave propagation.

\section{INTRODUCTION}

Determination of dynamic soil characteristics of shallow soil layers is important for the study of different problems in civil and geophysical engineering, such as ground borne vibrations or site amplification (Kausel \& Assimaki, 2002). Dynamic soil properties can be determined using laboratory and in situ methods. In situ methods have the advantage of not disturbing the soil

\footnotetext{
* Corresponding author: ramses.verachtert@kuleuven.be, tel: +32 16322231 Postprint submitted to Geophysical Journal International

Published version: R. Verachtert, G. Lombaert and G. Degrande. Determination of Rayleigh dispersion and attenuation curves Geophysical Journal International, 212:2143-2158, 2018 http://dx.doi.org/10.1093/gji/ggx523
} 
and examining large volumes of soil, in contrast to laboratory methods, which are susceptible to local variations of soil properties. Among the most popular in situ test methods are the surface wave experiments, which are simpler to perform and less expensive than borehole methods. Active Multichannel Analysis of Surface Waves (MASW) methods (Park et al., 1999; Foti et al., 2011) are often used to determine (multi-)modal Rayleigh dispersion and attenuation curves, which can be inverted to determine the shear wave velocity and material damping ratio profile of the soil. The solution of the inverse problem, however, is non-unique due to the limited identified frequency range. In order to reduce non-uniqueness, multi-modal inversion of dispersion and attenuation curves is preferred above single mode inversion (Xia et al., 2003; Socco \& Strobbia, 2004).

Many approaches have been developed to estimate multi-modal surface wave dispersion curves (Socco et al., 2010). These dispersion curves are often determined by transforming the measured vibrations from the time-space domain to the frequency-wavenumber (fk)-domain and picking the maxima (Park et al., 1999; Socco \& Strobbia, 2004). However, most of these transformation methods suffer from near field effects (Zywicki \& Rix, 2005). Zywicki and Rix (2005) identify two sources of near field effects: the presence of body wave propagation, which has a significant effect on the near wavefield and model incompatibility due to the use of transformation methods which assume plane waves, while the wavefield generated by a point source is cylindrical. Both near field effects distort the estimates of the Rayleigh phase velocity. As the signal-to-noise ratio is highest in the near field, it remains preferable to include near field information in the inversion process. This can be accomplished with an appropriate wave field transformation that accounts for the cylindrical symmetry of the spreading wave field, such as the cylindrical beamformer (Zywicki \& Rix, 2005) or a Hankel transformation (Forbriger, 2003; Badsar et al., 2010). Body waves, however, still influence the location of the Rayleigh wave poles in the fk-spectrum. This problem can be solved by defining the theoretical dispersion curves as the local maxima of the analytical fk-spectrum of the soil's Green's function.

Most methods for the determination of attenuation curves are based on spatial decay measurements of surface waves and assume that a single Rayleigh wave mode is dominant. They may lead to incorrect results if multiple modes significantly contribute to the wave field (Lai et al., 2002; Foti, 2004; Xia et al., 2002). In order to account for multiple modes, Badsar et al. (2010) proposed the half-power bandwidth method, which determines the attenuation of the Rayleigh wave modes based on the width of the Rayleigh peaks in the fk-domain. In the case of a single dominant Rayleigh wave mode, this method is as accurate as methods based on the spatial decay of surface waves; it is more accurate when multiple modes contribute significantly to the wave field. Misbah \& Strobbia (2014) recently proposed a method for estimating the complex wavenumbers of multiple modes, which can be considered as a high resolution subspace-based method for arbitrary receiver layout and source shot positions (Foti et al., 2014). The estimated attenuation coefficients, however, can still be influenced by interaction of different modes and event identification remains important.

In this paper, the circle fit method, originally developed to determine eigenfrequencies and modal damping ratios in structural dynamics (Ewins, 1984), is used to determine multi-modal Rayleigh dispersion and attenuation curves. Results are compared with the peak picking method and the half-power bandwidth method. It is shown that the circle fit method is not influenced by near field effects and less sensitive to mode interaction.

Wave propagation in a multi-layered halfspace with dynamic soil characteristics of a site in Lincent (Belgium), which is used as a benchmark, is discussed. Determination of Rayleigh dispersion and attenuation curves with the peak picking method and the half-power bandwidth method is briefly reviewed. The circle fit method is subsequently introduced as an alternative for the determination of multi-modal dispersion and attenuation curves.

Next, a MASW experiment is simulated for a site with a regular velocity profile to compare 
the results obtained with the circle fit method, the peak picking method and the half-power bandwidth method. Leakage and aliasing effects due to the truncation and discretization of the Hankel transform are discussed. This is followed by a similar analysis for a site with a soft layer trapped between two stiffer layers, in which case the peaks in the fk-spectrum are due to different higher order Rayleigh wave modes corresponding to an apparent phase velocity and attenuation coefficient. It is investigated how the results obtained with the circle fit method are affected by this velocity inversion.

Finally, the circle fit method, and a combination of the peak picking method and the half-power bandwidth method, are applied to determine experimental dispersion and attenuation curves from a MASW experiment at a site in Heverlee, Belgium. Both profiles are used to simulate the forcevelocity transfer functions and corresponding fk-spectra, that are subsequently compared with the experimental results.

\section{METHODOLOGY}

\subsection{Wave propagation in layered media}

Wave propagation in layered media can be studied with the direct stiffness method (Kausel \& Roësset, 1981) in the fk-domain, as implemented in the ElastoDynamics Toolbox (EDT) (Schevenels et al., 2009). The displacements $\tilde{\boldsymbol{U}}\left(k_{r}, \omega\right)$ are related to the tractions $\tilde{\boldsymbol{P}}\left(k_{r}, \omega\right)$ by:

$\tilde{\boldsymbol{K}}\left(k_{r}, \omega\right) \tilde{\boldsymbol{U}}\left(k_{r}, \omega\right)=\tilde{\boldsymbol{P}}\left(k_{r}, \omega\right)$.

The stiffness matrix $\tilde{\boldsymbol{K}}\left(k_{r}, \omega\right)$ is a function of the frequency $\omega$, the radial wavenumber $k_{r}$ and the following dynamic soil characteristics of each layer: the shear wave velocity $C_{\mathrm{s}}$, the dilatational wave velocity $C_{\mathrm{p}}$, the material damping ratios $\beta_{\mathrm{s}}$ and $\beta_{\mathrm{p}}$ in deviatoric and volumetric deformation, the density $\rho$ and the thickness $d$. Analytical expressions for the stiffness matrices of a halfspace and a layer element were derived by Kausel \& Roësset (1981). Material damping is assumed to be rate independent in the low frequency range and can be accounted for by applying the correspondence principle, valid for small damping ratios (Rizzo \& Shippy, 1971). This results in the use of complex Lamé coefficients $\mu\left(1+2 \beta_{\mathrm{s}} \mathrm{i}\right)$ and $(\lambda+2 \mu)\left(1+2 \beta_{\mathrm{p}} \mathrm{i}\right)$.

Dilatational waves (P-waves) and vertically polarized shear waves (SV-waves) are uncoupled from horizontally polarized shear waves (SH-waves). Relation (1) is therefore decomposed as:

$$
\begin{aligned}
\tilde{\boldsymbol{K}}_{\mathrm{P}-\mathrm{SV}}\left(k_{r}, \omega\right) \tilde{\boldsymbol{U}}_{\mathrm{P}-\mathrm{SV}}\left(k_{r}, \omega\right) & =\tilde{\boldsymbol{P}}_{\mathrm{P}-\mathrm{SV}}\left(k_{r}, \omega\right), \\
\tilde{\boldsymbol{K}}_{\mathrm{SH}}\left(k_{r}, \omega\right) \tilde{\boldsymbol{U}}_{\mathrm{SH}}\left(k_{r}, \omega\right) & =\tilde{\boldsymbol{P}}_{\mathrm{SH}}\left(k_{r}, \omega\right) .
\end{aligned}
$$

where the stiffness matrix $\tilde{\boldsymbol{K}}_{\mathrm{P}-\mathrm{SV}}\left(k_{r}, \omega\right)$, displacement vector $\tilde{\boldsymbol{U}}_{\mathrm{P}-\mathrm{SV}}\left(k_{r}, \omega\right)$ and traction vector $\tilde{\boldsymbol{P}}_{\mathrm{P}-\mathrm{SV}}\left(k_{r}, \omega\right)$ model P-SV wave propagation and the stiffness matrix $\tilde{\boldsymbol{K}}_{\mathrm{SH}}\left(k_{r}, \omega\right)$, displacement vector $\tilde{\boldsymbol{U}}_{\mathrm{SH}}\left(k_{r}, \omega\right)$ and traction vector $\tilde{\boldsymbol{P}}_{\mathrm{SH}}\left(k_{r}, \omega\right)$ model SH wave propagation.

If the traction vector $\tilde{\boldsymbol{P}}_{\mathrm{P}-\mathrm{SV}}\left(k_{r}, \omega\right)$ in equation (2) corresponds to a vertical Dirac impulse in space and time, the fundamental solution or Green's function $\tilde{u}_{z i}^{\mathrm{G}}\left(k_{r}, z, \omega\right)$ is obtained, where the first index $z$ refers to the loading direction and the second index $i$ to the displacement component. In this paper, Green's functions will primarily be evaluated along the surface $z=0$ and the dependence on the vertical coordinate $z$ will be omitted.

Table 1 presents the dynamic soil characteristics of a site in Lincent (Belgium) situated along the high speed line L2 between Brussels and Köln (Badsar et al., 2010). This site consists of a silt layer, followed by a layer of fine sand on top of a sequence of stiff layers of arenite and clay. Dynamic soil characteristics were determined by means of Spectral Analysis of Surface Wave (SASW) tests, Seismic Cone Penetration tests (SCPT) and seismic refraction tests (Schevenels 
Table 1. Dynamic soil characteristics of the site in Lincent (Belgium).

\begin{tabular}{cccccc}
\hline Layer & $\begin{array}{c}d \\
{[\mathrm{~m}]}\end{array}$ & $\begin{array}{c}C_{\mathrm{s}} \\
{[\mathrm{m} / \mathrm{s}]}\end{array}$ & $\begin{array}{c}C_{\mathrm{p}} \\
{[\mathrm{m} / \mathrm{s}]}\end{array}$ & $\begin{array}{c}\beta_{\mathrm{s}}=\beta_{\mathrm{p}} \\
{[-]}\end{array}$ & $\begin{array}{c}\rho \\
{\left[\mathrm{kg} / \mathrm{m}^{3}\right]}\end{array}$ \\
\hline 1 & 1.4 & 128 & 286 & 0.044 & 1900 \\
2 & 2.7 & 176 & 286 & 0.038 & 1900 \\
3 & $\infty$ & 355 & 1667 & 0.037 & 1900 \\
\hline
\end{tabular}

et al., 2008; Karl, 2005; Karl et al., 2006). This site is represented as a horizontally layered halfspace with two layers on top of a halfspace; the increase of the dilatational wave velocity in the halfspace is most probably due to saturation of the soil. This soil profile is subsequently used to illustrate the presented

Figure 1a shows the modulus of the normalized vertical Green's function $\overline{\tilde{u}}_{z z}^{\mathrm{G}}\left(k_{r}, \omega\right)$ as a function of the frequency $\omega$ and the phase velocity $C_{r}=\omega / k_{r}$ along the surface of the horizontally layered halfspace corresponding to the site in Lincent (table 1). The normalized simulated Green's function $\overline{\tilde{u}}_{z z}^{\mathrm{GS}}\left(k_{r}, \omega\right)$ presented in figure $1 \mathrm{~b}$ will be discussed in subsection 3.1.1. The Green's function $\tilde{u}_{z z}^{\mathrm{G}}\left(k_{r}, \omega\right)$ is normalized using the maximum modulus occuring at each frequency. The peaks in this spectrum correspond to Rayleigh waves or free surface waves with complex wavenumbers $k_{\mathrm{R} j}(\omega)$ that are solutions of the following eigenvalue problem:

$\operatorname{det} \tilde{\boldsymbol{K}}_{\mathrm{P}-\mathrm{SV}}\left(k_{r}, \omega\right)=0$.

The roots $k_{\mathrm{R} j}(\omega)$ can be determined by a search algorithm that minimizes the determinant in terms of the complex wavenumber $k_{r}$. The surface motion of a propagating Rayleigh wave at frequency $\omega$ is proportional to a zero-th order Hankel function of the second kind (Aki \& Richards, 2002):

$u_{z z}(r, t) \propto \operatorname{Re}\left(H_{0}^{(2)}\left(k_{\mathrm{R} j}(\omega) r\right) e^{+\mathrm{i} \omega t}\right)$.

The far field approximation of this expression is (Aki \& Richards, 2002):

$u_{z z}(r, t) \propto \operatorname{Re}\left(\mathrm{e}^{+\mathrm{i}\left(\omega t-k_{\mathrm{R} j}(\omega) r+\pi / 4\right)} / \sqrt{\left|k_{\mathrm{R} j}(\omega) r\right|}\right)$.

The theoretical Rayleigh phase velocity of the $j$-th mode is obtained as $C_{\mathrm{R} j}^{\mathrm{T}}(\omega)=\omega / \operatorname{Re}\left(k_{\mathrm{R} j}(\omega)\right)$. Figures 1a and 2a show the dispersion curves of the first four modes. $C_{\mathrm{R} 1}^{\mathrm{T}}(\omega)$ varies from the Rayleigh phase velocity of the underlying halfspace at limiting low frequencies to the Rayleigh phase velocity of the top layer at limiting high frequencies. The Rayleigh phase velocity of the higher wave modes varies from the shear wave velocity of the underlying halfspace to the shear wave velocity of the top layer at limiting high frequencies. The cut-on frequencies of the second, third and fourth Rayleigh mode are $14 \mathrm{~Hz}, 24 \mathrm{~Hz}$ and $48 \mathrm{~Hz}$, respectively. The theoretical dispersion curves $C_{\mathrm{R} j}^{\mathrm{Tp}}(\omega)$ and $C_{\mathrm{R} j}^{\mathrm{Tc}}(\omega)$ are determined with the peak picking and circle fit method, respectively, and will be discussed in subsection 2.2.1.

Attenuation of the Rayleigh waves is due to geometrical and material damping. The geometrical or radiation attenuation, due to wavefront spreading, is proportional to $r^{-1 / 2}$. Material damping is related to the dissipation of energy. The theoretical Rayleigh attenuation coefficient representing material damping of mode $j$ is calculated as $A_{\mathrm{R} j}^{\mathrm{T}}(\omega)=-\operatorname{Im}\left(k_{\mathrm{R} j}(\omega)\right)$. Figure $2 \mathrm{~b}$ shows the attenuation curves of the first four modes. At low frequencies, $A_{\mathrm{R} 1}^{\mathrm{T}}(\omega)$ is mainly determined by the material damping of the underlying halfspace. At higher frequencies, $A_{\mathrm{R} 1}^{\mathrm{T}}(\omega)$ is more influenced by the top layers. The energy dissipation of higher Rayleigh modes in the different soil layers is more difficult to predict, such that attenuation is not strictly increasing when the material damping ratios in the top layers are higher than in the underlying layers. The theoretical attenuation 

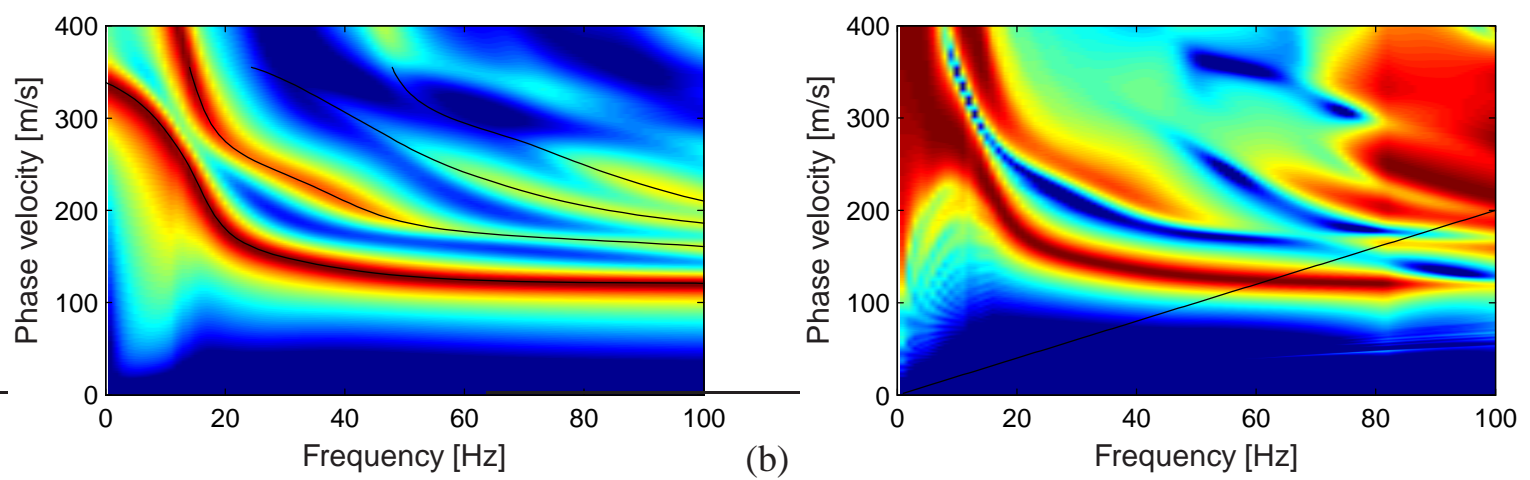

(a)

(b)

Figure 1. Fk-spectrum of (a) the normalized analytical Green's function $\overline{\tilde{u}}_{z z}^{\mathrm{G}}\left(k_{r}, \omega\right)$ with Rayleigh dispersion curves $C_{\mathrm{R} j}^{\mathrm{T}}(\omega)(j=1, \ldots, 4)$ (black lines) and (b) the normalized simulated Green's function $\overline{\tilde{u}}_{z z}^{\mathrm{GS}}\left(k_{r}, \omega\right)$ with spatial aliasing limit (black line).

curves $A_{\mathrm{R} j}^{\mathrm{Th}}(\omega)$ and $A_{\mathrm{R} j}^{\mathrm{Tc}}(\omega)$ are determined with the half-power bandwidth and circle fit method, respectively, and will be discussed in subsection 2.2.2.

\subsection{Frequency-wavenumber analysis of the Green's function}

Alternatively to solving the eigenvalue problem (4), the Rayleigh dispersion and attenuation curves can be determined at lower computational cost by analysis of the Green's function $\tilde{u}_{z z}^{\mathrm{G}}\left(k_{r}, \omega\right)$ in the frequency-wavenumber domain, as the location and width of the peaks in figure 1a are determined by the Rayleigh phase velocity and attenuation coefficient, respectively.

\subsubsection{Determination of the Rayleigh phase velocity}

In order to determine the Rayleigh phase velocity, assumptions are made regarding the shape of the Rayleigh peak. Badsar et al. (2010) determine the Rayleigh attenuation coefficients from the width of the Rayleigh peaks using the half-power bandwidth method. The underlying assumption for this method is that, in the case of hysteretic material damping, the peaks of the Green's function $\tilde{u}_{z z j}^{\mathrm{G}}\left(k_{r}, \omega\right)$ in the vicinity of each Rayleigh wavenumber $\operatorname{Re}\left(k_{\mathrm{R} j}(\omega)\right)$ can be approximated as

(a)

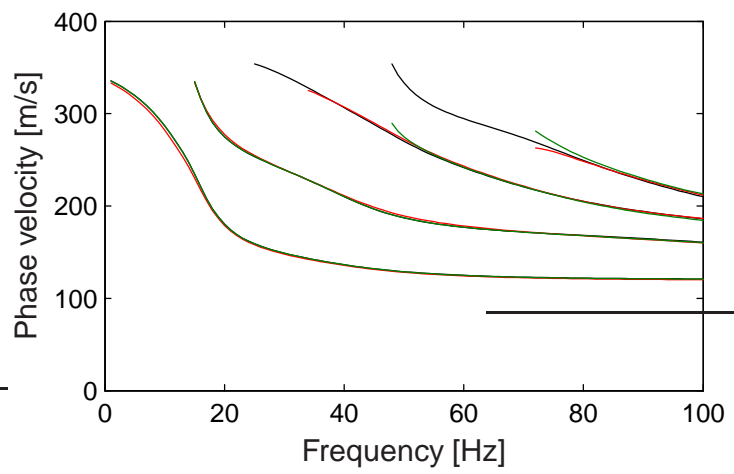

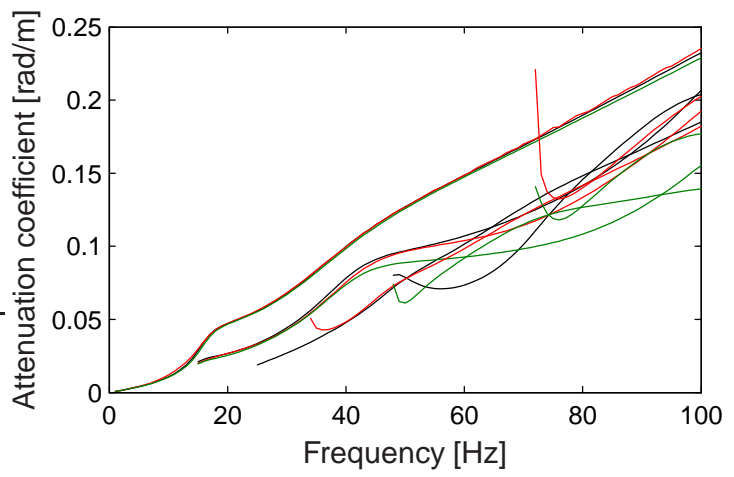

Figure 2. Rayleigh (a) dispersion curves $C_{\mathrm{R} j}^{\mathrm{T}}(\omega)(j=1,2,3,4)$ (black lines), $C_{\mathrm{R} j}^{\mathrm{Tp}}(\omega)$ (red lines) and $C_{\mathrm{R} j}^{\mathrm{Tc}}(\omega)$ (green lines) and (b) attenuation curves $A_{\mathrm{R} j}^{\mathrm{T}}(\omega)$ (black lines), $A_{\mathrm{R} j}^{\mathrm{Th}}(\omega)$ (red lines) and $A_{\mathrm{R} j}^{\mathrm{Tc}}(\omega)$ (green lines). 
(a)

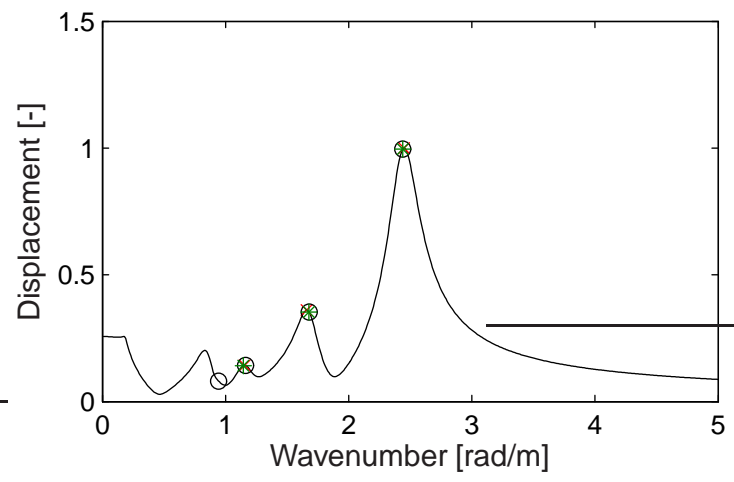

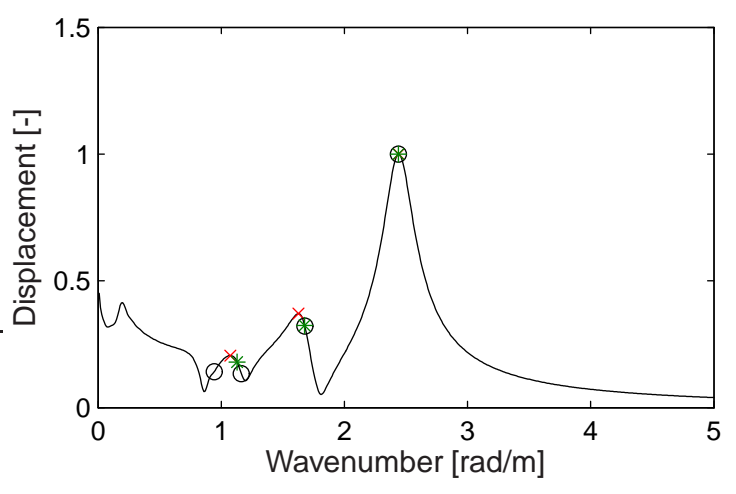

Figure 3. Modulus of (a) the normalized analytical Green's function $\overline{\tilde{u}}_{z z}^{\mathrm{G}}\left(k_{r}, \omega\right)$ (black line) at $50 \mathrm{~Hz}$ and $\overline{\tilde{u}}_{z z}^{\mathrm{G}}\left(\operatorname{Re}\left(k_{\mathrm{R} j}^{\mathrm{T}}(\omega)\right), \omega\right)(j=1,2,3,4)$ (black circles), $\overline{\tilde{u}}_{z z}^{\mathrm{G}}\left(k_{\mathrm{R} j}^{\mathrm{Tp}}(\omega), \omega\right)$ (red crosses) and $\overline{\tilde{u}}_{z z}^{\mathrm{G}}\left(k_{\mathrm{R} j}^{\mathrm{Tc}}(\omega), \omega\right)$ (green asterisks) and (b) the normalized simulated Green's function $\overline{\tilde{u}}_{z z}^{\mathrm{GS}}\left(k_{r}, \omega\right)$ (black line) at $50 \mathrm{~Hz}$ and $\overline{\tilde{u}}_{z z}^{\mathrm{GS}}\left(\operatorname{Re}\left(k_{\mathrm{R} j}^{\mathrm{T}}(\omega)\right), \omega\right)$ (black circles), $\overline{\tilde{u}}_{z z}^{\mathrm{GS}}\left(k_{\mathrm{R} j}^{\mathrm{Sp}}(\omega), \omega\right)$ (red crosses) and $\overline{\tilde{u}}_{z z}^{\mathrm{GS}}\left(k_{\mathrm{R} j}^{\mathrm{Sc}}(\omega), \omega\right)$ (green asterisks).

follows as a function of the real wavenumber $k_{r}$ :

$\tilde{u}_{z z j}^{\mathrm{G}}\left(k_{r}, \omega\right) \simeq \frac{C_{1 j}(\omega)}{\beta_{j}^{2}-1+\mathrm{i} \alpha_{j}}+C_{2 j}(\omega)$,

where $\beta_{j}=k_{r} / \operatorname{Re}\left(k_{\mathrm{R} j}(\omega)\right)$ and $\alpha_{j}=2 A_{\mathrm{R} j}(\omega) / \operatorname{Re}\left(k_{\mathrm{R} j}(\omega)\right)$. The complex mode dependent constants $C_{1 j}(\omega)$ and $C_{2 j}(\omega)$ are introduced to obtain a better approximation of the Green's function, as will be explained in the following. The range of wavenumbers for which only the first term in equation (7) is a good approximation depends on the separation of the different Rayleigh peaks and the interaction with different wave modes. For dominant Rayleigh waves, $C_{2 j}(\omega)$ is small compared to the peak amplitude of $\tilde{u}_{z z j}^{\mathrm{G}}\left(k_{r}, \omega\right)$ and a good estimate $k_{\mathrm{R} j}^{\mathrm{Tp}}(\omega)$ of the real part of $k_{\mathrm{R} j}^{\mathrm{T}}(\omega)$ can be found by peak picking.

Figure 3a shows the modulus of the normalized analytical Green's function $\overline{\tilde{u}}_{z z}^{\mathrm{G}}\left(k_{r}, \omega\right)$ at $50 \mathrm{~Hz}$ and the locations of the Rayleigh modes determined by the peak picking method. The largest peak in figure 3a corresponds to the fundamental Rayleigh mode. The other peaks at smaller wavenumbers correspond to higher Rayleigh modes. The Rayleigh wavenumber estimates of the first three modes are very accurate and the markers indicating the wavenumbers $k_{\mathrm{R} j}^{\mathrm{Tp}}(\omega)$ and $k_{\mathrm{R} j}^{\mathrm{T}}(\omega)$ perfectly overlap. The fourth mode could not be identified, as there is no peak corresponding to $\operatorname{Re}\left(k_{\mathrm{R} 4}^{\mathrm{T}}(\omega)\right.$ ). The peak at $k_{r}=0.83 \mathrm{rad} / \mathrm{m}$ actually corresponds to the fifth mode. Peak picking is performed for the first four Rayleigh modes for frequencies up to $100 \mathrm{~Hz}$. The normalized simulated Green's function $\overline{\tilde{u}}_{z z}^{\mathrm{GS}}\left(k_{r}, \omega\right)$ presented in figure $3 \mathrm{~b}$ will be discussed in subsection 3.1.1. Figure 2a compares the theoretical dispersion curves $C_{\mathrm{R} j}^{\mathrm{Tp}}(\omega)=\omega / k_{\mathrm{R} j}^{\mathrm{Tp}}(\omega)$, determined with the peak picking method, with $C_{\mathrm{R} j}^{\mathrm{T}}(\omega)$. The first two modes are accurately identified in the considered frequency range. The peak picking method could only identify the third mode for frequencies above $34 \mathrm{~Hz}$ and converged to other modes below this frequency because the Rayleigh peak is too small to be identified. Similarly, the fourth mode could only be identified for frequencies above $72 \mathrm{~Hz}$.

If only the modulus of the Green's function is considered, valuable information related to the phase is lost. Following theoretical developments of Ewins (1984) in structural dynamics to determine eigenfrequencies and modal damping ratios, the circle fit method is presented to determine the Rayleigh wavenumber. Figure 4 shows a Nyquist plot of the Green's function $\tilde{u}_{z z}^{\mathrm{G}}\left(k_{r}, \omega\right)$ at $50 \mathrm{~Hz}$ for 500 equidistant wavenumbers from $k_{r}=0.01 \mathrm{rad} / \mathrm{m}$ to $k_{r}=5 \mathrm{rad} / \mathrm{m}$, mapping its imaginary part against its real part in the frame of reference Re - Im. It is observed that the different Rayleigh peaks correspond to circles. If the original frame of reference $\mathrm{Re}-\mathrm{Im}$ is translated (sub- 


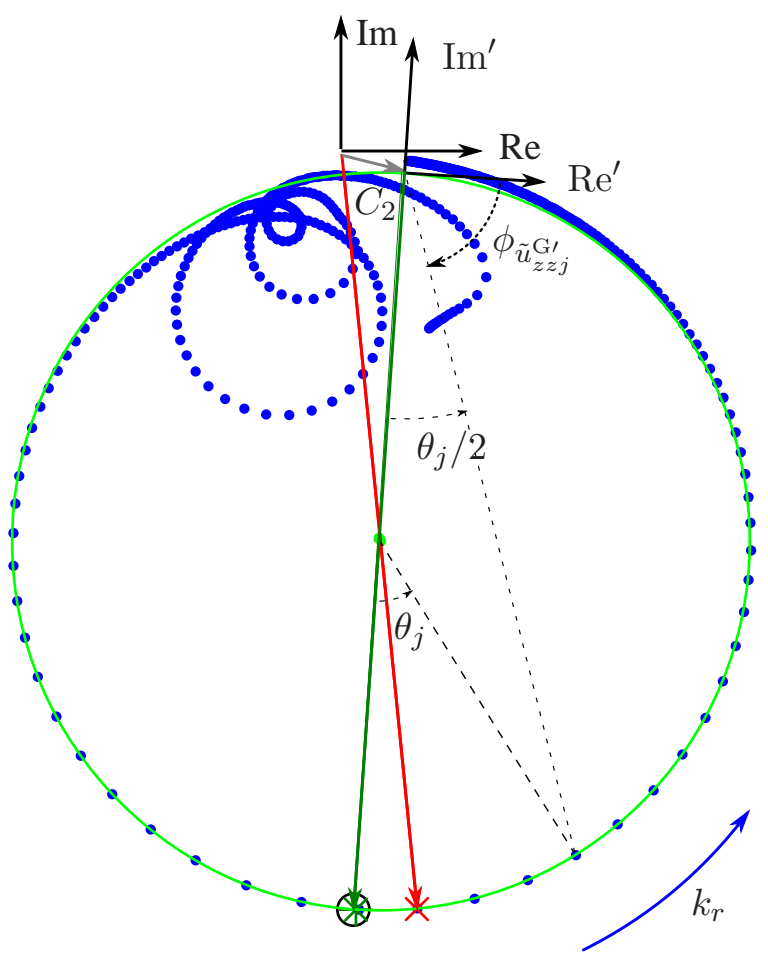

Figure 4. Nyquist plot of the Green's function $\tilde{u}_{z z}^{\mathrm{G}}\left(k_{r}, \omega\right)$ at $50 \mathrm{~Hz}$ (blue dots), fitted circle for the first Rayleigh wave mode (green line), and $\tilde{u}_{z z}^{\mathrm{G}}\left(\operatorname{Re}\left(k_{\mathrm{R} 1}^{\mathrm{T}}(\omega)\right), \omega\right)$ (black circle), $\tilde{u}_{z z}^{\mathrm{G}}\left(k_{\mathrm{R} 1}^{\mathrm{Tp}}(\omega), \omega\right)$ (red cross) and $\tilde{u}_{z z}^{\mathrm{G}}\left(k_{\mathrm{R} 1}^{\mathrm{Tc}}(\omega), \omega\right)$ (green asterisk).

tracting $C_{2 j}(\omega)$ in equation (7)) and rotated (dividing by $C_{1 j}(\omega)$ in equation (7)) to the frame of reference $\mathrm{Re}^{\prime}-\mathrm{Im}^{\prime}$, the approximation of the Green's function $\tilde{u}_{z z j}^{\mathrm{G} \prime}\left(k_{r}, \omega\right)$ in the vicinity of the Rayleigh wavenumber $\operatorname{Re}\left(k_{\mathrm{R} j}(\omega)\right)$ can be written as:

$\tilde{u}_{z z j}^{\mathrm{G} \prime}\left(k_{r}, \omega\right) \simeq \frac{1}{\beta_{j}^{2}-1+\mathrm{i} \alpha_{j}}$,

which is equivalent to introducing complex parameters $C_{1 j}(\omega)=1$ and $C_{2 j}(\omega)=0$ in equation (7). It can be demonstrated that the real and imaginary part of $\tilde{u}_{z z j}^{\mathrm{G} \prime}\left(k_{r}, \omega\right)$ are related as:

$\operatorname{Re}\left(\tilde{u}_{z z j}^{\mathrm{G} \prime}\left(k_{r}, \omega\right)\right)^{2}+\left(\operatorname{Im}\left(\tilde{u}_{z z j}^{\mathrm{G} \prime}\left(k_{r}, \omega\right)\right)+\frac{1}{2 \alpha_{j}}\right)^{2}=\left(\frac{1}{2 \alpha_{j}}\right)^{2}$,

which is the equation of a circle with centre $\left(0,-\mathrm{i} /\left(2 \alpha_{j}\right)\right)$ on the imaginary axis and radius $1 /\left(2 \alpha_{j}\right)$, hence the name of the method.

It is observed in figure 4 that the spacing between the different points is largest in the vicinity of $\tilde{u}_{z z}^{\mathrm{G}}\left(\operatorname{Re}\left(k_{\mathrm{R} 1}^{\mathrm{T}}(\omega)\right), \omega\right)$. This is equivalent to stating that the angular sweep $\partial \theta_{j} / \partial k_{r}$ of the central angle $\theta_{j}$, subtending the arc between $\tilde{u}_{z z j}^{\mathrm{G} \prime}\left(k_{r}, \omega\right)$ and the negative $\mathrm{Im}^{\prime}$-axis, attains a maximum at $\operatorname{Re}\left(k_{\mathrm{R} 1}^{\mathrm{T}}(\omega)\right)$. This property is proven in the following. In order to express $\theta_{j}$ as a function of the wavenumber $k_{r}$, the phase $\phi_{\tilde{u}_{z z j}^{\mathrm{G} \prime}}$ of the Green's function is related to the central angle $\theta_{j}$ (figure 4):

$\phi_{\tilde{u}_{z z j}^{\mathrm{G} \prime}}=\frac{\theta_{j}}{2}-\frac{\pi}{2}$. 
It follows that:

$\tan \left(\frac{\theta_{j}}{2}\right)=-\cot \left(\phi_{\tilde{u} \tilde{u}_{z z j}^{\mathrm{G} \prime}}\right)=-\frac{\operatorname{Re}\left(\tilde{u}_{z z j}^{\mathrm{G} \prime}\left(k_{r}, \omega\right)\right)}{\operatorname{Im}\left(\tilde{u}_{z z j}^{\mathrm{G} \prime}\left(k_{r}, \omega\right)\right)}=\frac{\beta_{j}^{2}-1}{\alpha_{j}}$

so that:

$\theta_{j}=2 \arctan \left(\frac{\beta_{j}^{2}-1}{\alpha_{j}}\right)$.

Differentiation with respect to $\beta_{j}$ results in:

$$
\frac{\partial \theta_{j}}{\delta \beta_{j}}=\frac{4 \beta_{j}}{\alpha_{j}\left[1+\left(\frac{\beta_{j}^{2}-1}{\alpha_{j}}\right)^{2}\right]},
$$

which attains a maximum at $\beta_{j}=1.001$ for a realistic value $\alpha_{j}=0.1$. For a relatively large value $\alpha_{j}=0.4$, the maximum is obtained at $\beta_{j}=1.02$, which is still relatively close to 1 . As $\beta_{j}$ is defined as $k_{r} / \operatorname{Re}\left(k_{\mathrm{R} j}(\omega)\right)$, the angular sweep $\partial \theta_{j} / \partial k_{r}$ attains a maximum at $k_{r}=\operatorname{Re}\left(k_{\mathrm{R} j}(\omega)\right)$. The bias introduced by the circle fit method is very small and not influenced by the transformation from $\tilde{u}_{z z j}^{\mathrm{G}}\left(k_{r}, \omega\right)$ to $\tilde{u}_{z z j}^{\mathrm{G} \prime}\left(k_{r}, \omega\right)$, or independent of the complex constants $C_{1 j}(\omega)$ and $C_{2 j}(\omega)$, as opposed to the peak picking method. Therefore, the circle fit method is proposed in this paper to estimate the real part of $k_{\mathrm{R} j}(\omega)$, without prior knowledge of $C_{1 j}(\omega)$ and $C_{2 j}(\omega)$.

In appendix A, a search algorithm is described to determine the circle fit estimate $k_{\mathrm{R} j}^{\mathrm{Tc}}(\omega)$ and the corresponding theoretical Rayleigh phase velocity $C_{\mathrm{R} j}^{\mathrm{Tc}}(\omega)=\omega / k_{\mathrm{R} j}^{\mathrm{Tc}}(\omega)$. Computation of the complex constants $C_{1 j}(\omega)$ and $C_{2 j}(\omega)$ gives no benefit when calculating the dispersion curves and is therefore not performed. As can be noticed on figure $4, \tilde{u}_{z z}^{\mathrm{G}}\left(k_{\mathrm{R} 1}^{\mathrm{Tc}}(\omega), \omega\right)$ perfectly corresponds with $\tilde{u}_{z z}^{\mathrm{G}}\left(k_{\mathrm{R} 1}^{\mathrm{T}}(\omega), \omega\right)$.

The fitted circles in figure 5a correspond to the Rayleigh peaks in figure 3a of the first, second and third mode. There is no circle corresponding to the fourth mode. The smaller circles, corresponding to higher Rayleigh wave modes, are shifted further away from the origin than the circle corresponding to the first mode, relatively to their radius, resulting in less accurate estimates $k_{\mathrm{R} j}^{\mathrm{Tp}}(\omega)$ obtained with the peak picking method. The estimates $k_{\mathrm{R} j}^{\mathrm{Tc}}(\omega)$ obtained with the circle fit method are not affected by the shifting of the circles and the markers indicating the wavenumbers $k_{\mathrm{R} j}^{\mathrm{Tc}}(\omega)$ and $k_{\mathrm{R} j}^{\mathrm{T}}(\omega)$ perfectly overlap in figures 3a and 5a. The normalized simulated Green's function $\overline{\tilde{u}}_{z z}^{\mathrm{GS}}\left(k_{r}, \omega\right)$ presented in figure $5 \mathrm{~b}$ will be discussed in subsection 3.1.1.

Figure 2 a shows the estimates $C_{\mathrm{R} j}^{\mathrm{Tc}}(\omega)$ of the Rayleigh phase velocity obtained with the circle fit method, for the first four Rayleigh modes, for frequencies up to $100 \mathrm{~Hz}$. The first two modes are completely identified and the circle fit method is slightly more accurate than the peak picking method. The third and fourth mode are identified for frequencies above $48 \mathrm{~Hz}$ and $72 \mathrm{~Hz}$, respectively. For these modes, the peak picking method is slightly more accurate. This indicates that the Rayleigh peaks of these modes are influenced by other wave modes. Nevertheless, it is shown in the next section that the circle fit method is more robust when applied to a simulated surface wave experiment.

\subsubsection{Determination of the Rayleigh attenuation coefficient}

Badsar et al. (2010) propose a generalized formulation of the half-power bandwidth method to determine the Rayleigh attenuation coefficient $A_{\mathrm{R} j}^{\mathrm{Th}}(\omega)$ of different modes for the case of weak 

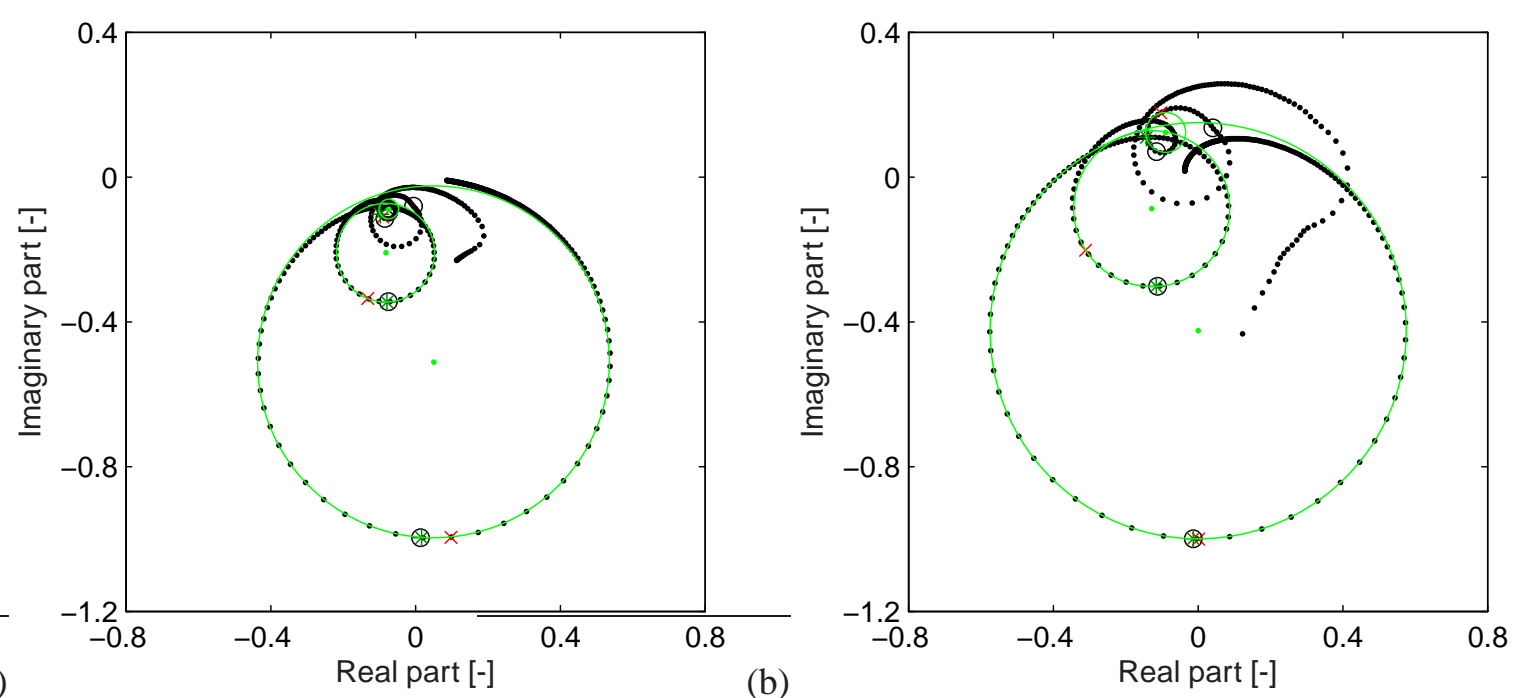

(a)

(b)

Figure 5. Nyquist plot and fitted circles (green lines) of (a) the normalized analytical Green's function $\overline{\tilde{u}}_{z z}^{\mathrm{G}}\left(k_{r}, \omega\right)$ at $50 \mathrm{~Hz}$ and $\overline{\tilde{u}}_{z z}^{\mathrm{G}}\left(\operatorname{Re}\left(k_{\mathrm{R} j}^{\mathrm{T}}(\omega)\right), \omega\right)(j=1,2,3,4)$ (black circles), $\overline{\tilde{u}}_{z z}^{\mathrm{G}}\left(k_{\mathrm{R} j}^{\mathrm{Tp}}(\omega), \omega\right)$ (red crosses) and $\overline{\tilde{u}}_{z z}^{\mathrm{G}}\left(k_{\mathrm{R} j}^{\mathrm{Tc}}(\omega), \omega\right)$ (green asterisks) and (b) the normalized simulated Green's function $\overline{\tilde{u}}_{z z}^{\mathrm{GS}}\left(k_{r}, \omega\right)$ at 50 $\mathrm{Hz}$ and $\overline{\tilde{u}}_{z z}^{\mathrm{GS}}\left(\operatorname{Re}\left(k_{\mathrm{R} j}^{\mathrm{T}}(\omega)\right), \omega\right)$ (black circles), $\overline{\tilde{u}}_{z z}^{\mathrm{GS}}\left(k_{\mathrm{R} j}^{\mathrm{Sp}}(\omega), \omega\right)$ (red crosses) and $\overline{\tilde{u}}_{z z}^{\mathrm{GS}}\left(k_{\mathrm{R} j}^{\mathrm{Sc}}(\omega), \omega\right)$ (green asterisks).

damping:

$A_{\mathrm{R} j}^{\mathrm{Th}}(\omega)=\frac{k_{r b}-k_{r a}}{2 \sqrt{\gamma^{-2}-1}}$

where $k_{r a}$ and $k_{r b}$ are wavenumbers smaller and larger than $k_{\mathrm{R} j}^{\mathrm{Tp}}(\omega)$, such that:

$\left|\tilde{u}_{z z}^{\mathrm{G}}\left(k_{r a}, \omega\right)\right|=\left|\tilde{u}_{z z}^{\mathrm{G}}\left(k_{r b}, \omega\right)\right|=\gamma\left|\tilde{u}_{z z}^{\mathrm{G}}\left(k_{\mathrm{R} j}^{\mathrm{Tp}}(\omega), \omega\right)\right|$.

For the classical choice of $\gamma=1 / \sqrt{2}, k_{r a}$ and $k_{r b}$ are the so-called half-power bandwidth points. Badsar et al. (2010) suggest to use $\gamma=0.99$ in order to avoid influence of neighbouring Rayleigh wave peaks. The method assumes that $C_{2 j}(\omega)$ in equation (7) equals zero, but also gives good estimates for small values of $C_{2 j}(\omega)$.

Figure $2 \mathrm{~b}$ shows the estimate of the attenuation coefficients $A_{\mathrm{R} j}^{\mathrm{Th}}(\omega)$ obtained with the halfpower bandwidth method, at the frequencies for which the corresponding modes were identified with the peak picking method. The attenuation coefficient $A_{\mathrm{R} 1}^{\mathrm{Th}}(\omega)$ of the first mode is accurately quantified in the frequency range considered, $A_{\mathrm{R} 2}^{\mathrm{Th}}(\omega)$ is accurate up to $60 \mathrm{~Hz}, A_{\mathrm{R} 3}^{\mathrm{Th}}(\omega)$ is accurate between $38 \mathrm{~Hz}$ and $60 \mathrm{~Hz}$, and $A_{\mathrm{R} 4}^{\mathrm{Th}}(\omega)$ is not accurate at all. At frequencies higher than $60 \mathrm{~Hz}$, the Rayleigh peaks corresponding to the higher modes are less accurate, as they are not sufficiently separated. In order to reduce the influence of interacting Rayleigh modes on the estimation of the attenuation coefficients, the circle fit method is alternatively proposed (Ewins, 1984).

For wavenumbers $k_{r a}$ and $k_{r b}$ in the vicinity of $k_{\mathrm{R} j}^{\mathrm{Tc}}(\omega)$ and $k_{r a}<k_{\mathrm{R} j}^{\mathrm{Tc}}(\omega)<k_{r b}$, the following expressions can be derived from equation (11):

$$
\begin{aligned}
& \tan \left(\left|\frac{\theta_{j}\left(k_{r a}, \omega\right)}{2}\right|\right)=\frac{1-\left(\frac{k_{r a}}{k_{\mathrm{R} j}^{T c}(\omega)}\right)^{2}}{\alpha_{j}}, \\
& \tan \left(\left|\frac{\theta_{j}\left(k_{r b}, \omega\right)}{2}\right|\right)=\frac{\left(\frac{k_{r b}}{k_{\mathrm{R} j}^{T c}(\omega)}\right)^{2}-1}{\alpha_{j}},
\end{aligned}
$$


where $\beta_{j}$ is replaced by the circle fit estimate $k_{r} / k_{\mathrm{R} j}^{\mathrm{Tc}}$. Summing both equations and replacing $\alpha_{j}=2 A_{\mathrm{R} j}^{\mathrm{Tc}}(\omega) / k_{\mathrm{R} j}^{\mathrm{Tc}}(\omega)$ results in the theoretical Rayleigh attenuation coefficient determined with the circle fit method:

$$
A_{\mathrm{R} j}^{\mathrm{Tc}}(\omega)=\frac{k_{r b}^{2}-k_{r a}^{2}}{2 k_{\mathrm{R} j}^{\mathrm{Tc}}(\omega)\left[\tan \left(\frac{\left|\theta_{j}\left(k_{r b}, \omega\right)\right|}{2}\right)+\tan \left(\frac{\left|\theta_{j}\left(k_{r a}, \omega\right)\right|}{2}\right)\right]} .
$$

If $k_{r a}$ and $k_{r b}$ are chosen such that $\left|\theta_{j}\left(k_{r a}, \omega\right)\right|=\left|\theta_{j}\left(k_{r b}, \omega\right)\right|=\pi / 2$, equation (18) reduces to $A_{\mathrm{R} j}^{\mathrm{Tc}}(\omega)=\left(k_{r b}^{2}-k_{r a}^{2}\right) /\left(4 k_{\mathrm{R} j}^{\mathrm{Tc}}(\omega)\right)$; for weak damping, this can be further simplified to $A_{\mathrm{R} j}^{\mathrm{Tc}}(\omega)=$ $\left(k_{r b}-k_{r a}\right) / 2$. This is equivalent to equation (14) for $\gamma=1 / \sqrt{2}$, in which case $k_{r a}$ and $k_{r b}$ are the half-power bandwidth points. The circle fit method is only equivalent to the half-power bandwidth method if the complex constant $C_{2 j}(\omega)$ equals 0 . As the central angle $\theta_{j}$ does not depend upon $C_{2 j}(\omega)$, the circle fit method is more robust than the half-power bandwidth method.

In order to fit a circle through the points corresponding to the Rayleigh peak, the width of the Rayleigh peak needs to be known. Sufficient points on the Rayleigh circle must be chosen in order to obtain an accurate fit, but the wavenumber range of these points cannot be too large in order to avoid influence of other wave modes. A bandwidth $\Delta k_{r}=A_{\mathrm{R} j}^{\mathrm{Th}}(\omega)$ is proposed. For a Rayleigh circle which passes through the origin, this bandwidth spans $30 \%$ of the circle, which is found to be adequate. Equation (14) can therefore be used to determine the width of the Rayleigh peak. Appendix B describes an algorithm to obtain the Rayleigh attenuation curves based on equation (18).

Figure $2 \mathrm{~b}$ shows the estimates of the attenuation coefficient $A_{\mathrm{R} j}^{\mathrm{Tc}}(\omega)$ obtained with the circle fit method, at the same frequencies for which the Rayleigh phase velocities were identified. The attenuation coefficient $A_{\mathrm{R} 1}^{\mathrm{Tc}}(\omega)$ of the first mode is accurately determined. $A_{\mathrm{R} 2}^{\mathrm{Tc}}(\omega)$ is accurate up to $35 \mathrm{~Hz}$, and $A_{\mathrm{R} 3}^{\mathrm{Tc}}(\omega)$ and $A_{\mathrm{R} 4}^{\mathrm{Tc}}(\omega)$ are not accurate. The attenuation coefficient determined with the half-power bandwidth method corresponds better to the theoretical attenuation coefficient for all modes. The attenuation coefficient determined with the circle fit method, however, is an unambiguously determined characteristic of the Rayleigh mode, which can be used in an inversion process to determine the material damping ratio profile of a site. Moreover, it is shown in the next section that the attenuation coefficient determined with the circle fit method is more robust and easier to identify than the attenuation coefficient determined with the half-power bandwidth method, especially for non-dominant Rayleigh waves. The attenuation coefficient determined with the circle fit method is thus more adequate for the multi-modal inversion of experimental attenuation curves.

\section{SIMULATED MASW EXPERIMENTS}

In an active MASW experiment, the soil is excited by a hammer impact, a drop weight or a shaker on a surface foundation, and the velocity or acceleration response is measured with different receivers (geophones or accelerometers) along a measurement line in the free field. From this response, an experimental fk-spectrum is obtained, which can subsequently be used to determine experimental estimates of Rayleigh dispersion and attenuation curves. In this section, MASW experiments are simulated considering impact loading on a layered halfspace with a regular velocity profile, corresponding to the site in Lincent introduced in the previous section, and a layered halfspace with an irregular velocity profile where a soft layer is trapped between two stiffer layers. The computed response allows to compare Rayleigh dispersion and attenuation curves determined by the circle fit method, the peak picking method and the half-power bandwidth method. 


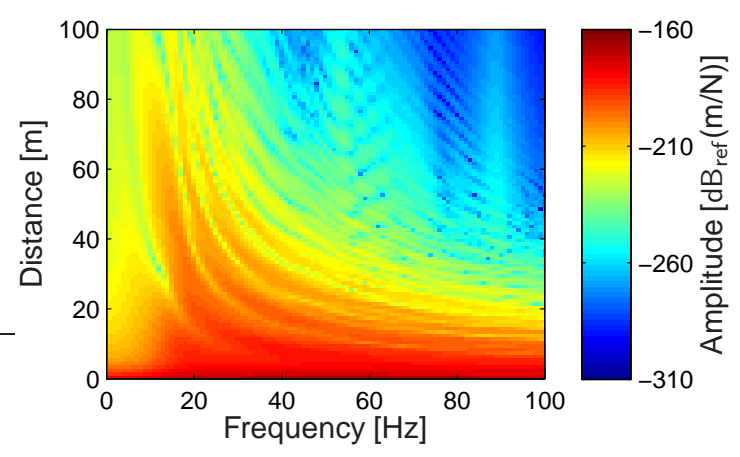

Figure 6. Amplitude of the simulated Green's function $\hat{u}_{z z}^{\mathrm{GS}}(r, \omega)$ for the site in Lincent.

\subsection{Site with a regular velocity profile}

\subsubsection{Simulated free field vibration}

A surface wave experiment is simulated by calculating the vibration response of a horizontally layered halfspace, corresponding to the site in Lincent (Belgium), due to a vertical impact at the surface. This impact is simulated by a Dirac impulse in space and time that is directly applied to the soil as it can be demonstrated that the effect of dynamic foundation-soil interaction is limited if the wavelengths in the soil are large compared to the dimensions of the foundation (Schevenels, 2007). The free field vibrations therefore correspond to the Green's functions. As demonstrated in the previous section, the Green's function $\tilde{u}_{z z}^{\mathrm{G}}\left(k_{r}, \omega\right)$ can be calculated analytically in the fkdomain. An inverse Hankel transform from the wavenumber to the spatial domain accounts for the cylindrical symmetry of the wavefronts:

$\hat{u}_{z z}^{\mathrm{G}}(r, \omega)=\int_{0}^{\infty} \tilde{u}_{z z}^{\mathrm{G}}\left(k_{r}, \omega\right) J_{0}\left(k_{r} r\right) k_{r} \mathrm{~d} k_{r}$.

This inverse Hankel transformation is performed by means of the ElastoDynamics Toolbox (EDT) for MATLAB (Schevenels et al., 2009). The Green's function $\tilde{u}_{z z}^{\mathrm{G}}\left(k_{r}, \omega\right)$ exhibits a first order singularity and is therefore decomposed into a singular part, which is transformed analytically, and a regular part, which is transformed numerically with 5000 wavenumbers, logarithmically sampled between $10^{-8} \omega$ and $10^{4} \omega[\mathrm{rad} / \mathrm{m}]$ for each frequency $\omega$. Figure 6 shows the resulting simulated vertical displacement $\hat{u}_{z z}^{\mathrm{GS}}(r, \omega)$ at the soil's surface, calculated for an array of 100 equidistant receivers located from $r_{\min }=1 \mathrm{~m}$ to $r_{\max }=100 \mathrm{~m}$ from the source with a receiver spacing $\Delta r=1 \mathrm{~m}$. The effect of material damping is larger at high frequencies or decreasing Rayleigh wavelength.

These samples are subsequently used in a truncated forward Hankel transform to obtain the simulated fk-spectrum $\tilde{u}_{z z}^{\mathrm{GS}}\left(k_{r}, \omega\right)$. The simulated fk-spectrum $\tilde{u}_{z z}^{\mathrm{GS}}\left(k_{r}, \omega\right)$ differs from the analytical fk-spectrum $\tilde{u}_{z z}^{\mathrm{G}}\left(k_{r}, \omega\right)$, as it is affected by the spatial sampling related to the receiver setup. Following Forbriger (2003), Badsar et al. (2010) replace the Bessel function $J_{0}\left(k_{r} r\right)$ by the zeroth order Hankel function of the first kind $H_{0}^{(1)}\left(k_{r} r\right) / 2$ to reduce aliasing by accounting for the fact that the wave field consists of outgoing waves only. The following transformation is obtained:

$\tilde{u}_{z z}^{\mathrm{GS}}\left(k_{r}, \omega\right)=\frac{1}{2} \int_{0}^{r_{\max }} \hat{u}_{z z}^{\mathrm{GS}}(r, \omega) H_{0}^{(1)}\left(k_{r} r\right) r \mathrm{~d} r$.

This integral is evaluated using a generalized Filon quadrature using linear interpolation (Frazer \& Gettrust, 1984). Due to the spatial sampling of $\hat{u}_{z z}^{\mathrm{GS}}(r, \omega)$ with receiver spacing $\Delta r$, the fkspectrum suffers from spatial aliasing for wavenumbers $k_{r}>\pi / \Delta r$. The simulated Green's function $\overline{\tilde{u}}_{z z}^{\mathrm{GS}}\left(k_{r}, \omega\right)$ is normalized so that the maximum modulus equals 1 at each frequency and is 
shown in figure $1 \mathrm{~b}$ as a function of the frequency and the phase velocity $C_{r}=\omega / k_{r}$. The equivalent spatial aliasing limit becomes $C_{r}^{\text {aliasing }}=\omega \Delta r / \pi$. At $62 \mathrm{~Hz}$, this aliasing limit crosses the dispersion curve of the fundamental mode, resulting in larger peaks for the higher modes, when compared with the analytical fk-spectrum.

Truncation of the Hankel transformation to $r_{\max }$ in equation (20) results in leakage and widening of the Rayleigh peaks, and thus an overestimation of the attenuation coefficient. Leakage mostly affects low frequencies were the attenuation coefficient is small. In order to reduce leakage, an exponentially decaying window $\hat{w}(r, \omega)=e^{-\hat{A}_{\text {art }}(\omega) r}$ is applied to the frequency-space domain data when calculating the attenuation coefficient (Badsar et al., 2010). A similar windowing technique is commonly used in structural dynamics to determine the damping ratio of weakly damped systems from a free vibration signal with limited duration (Fladung \& Rost, 1997). The application of an exponential window can be considered as the introduction of artificial damping, resulting in a stronger spatial decay of the surface waves. The decay rate is determined by $\hat{A}_{\text {art }}(\omega)$, the smallest positive value that satisfies the following inequality:

$\frac{\left|\hat{w}\left(r_{\max }, \omega\right) \hat{u}_{z z}^{\mathrm{GS}}\left(r_{\max }, \omega\right)\right|}{\left|\hat{w}\left(r_{\min }, \omega\right) \hat{u}_{z z}^{\mathrm{GS}}\left(r_{\min }, \omega\right)\right|} \leq q$

The application of the window ensures that the amplitude ratio of the response at the farthest and the nearest receiver does not exceed a value $q$. The optimal value of $q$ depends on the receiver setup. Badsar et al. (2010) recommend a value $q=10^{-4}$ for the setup used here.

The simulated fk-spectrum can subsequently be used to calculate the simulated Rayleigh phase velocities $C_{\mathrm{R} j}^{\mathrm{Sp}}(\omega)=\omega / k_{\mathrm{R} j}^{\mathrm{Sp}}(\omega)$ and $C_{\mathrm{R} j}^{\mathrm{Sc}}(\omega)=\omega / k_{\mathrm{R} j}^{\mathrm{Sc}}(\omega)$ with the peak picking method and the circle fit method, respectively. Similarly, the simulated attenuation coefficients $A_{\mathrm{R} j}^{\mathrm{Sh}}(\omega)$ and $A_{\mathrm{R} j}^{\mathrm{Sc}}(\omega)$ are determined with the half-power bandwidth method and the circle fit method, respectively, from the simulated fk-spectrum. Due to the use of the exponential window, $\hat{A}_{\text {art }}(\omega)$ should be subtracted from the obtained attenuation coefficient. No window function is used when determining the Rayleigh phase velocity.

\subsubsection{Rayleigh dispersion curves}

Figure $3 \mathrm{~b}$ shows the modulus of the normalized simulated Green's function $\overline{\tilde{u}}_{z z}^{\mathrm{GS}}\left(k_{r}, \omega\right)$ at 50 $\mathrm{Hz}$. The value of the Green's function at the real part $\operatorname{Re}\left(k_{\mathrm{R} j}^{\mathrm{T}}(\omega)\right)$ of the theoretical Rayleigh wavenumber and at the real wavenumbers $k_{\mathrm{R} j}^{\mathrm{Sp}}(\omega)$ and $k_{\mathrm{R} j}^{\mathrm{Sc}}(\omega)$, estimated by means of the peak picking method and the circle fit method, respectively, are indicated with markers. These markers perfectly overlap for the first Rayleigh mode. Due to the discretization and truncation of the wavenumber transformation (20), the Rayleigh peaks in the simulated Green's function $\overline{\tilde{u}}_{z z}^{\mathrm{GS}}\left(k_{r}, \omega\right)$ are no longer perfectly located at $\operatorname{Re}\left(k_{\mathrm{R} j}^{\mathrm{T}}(\omega)\right)$. The shift is negligible for the first Rayleigh mode, but becomes more apparent for the higher modes, resulting in a difference between $k_{\mathrm{R} j}^{\mathrm{Sp}}(\omega)$ and $\operatorname{Re}\left(k_{\mathrm{R} j}^{\mathrm{T}}(\omega)\right)$. This is explained by examining the Nyquist plot of $\overline{\tilde{u}}_{z z}^{\mathrm{GS}}\left(k_{r}, \omega\right)$ at $50 \mathrm{~Hz}$ (figure $5 \mathrm{~b}$ ). When compared to figure $5 \mathrm{a}$, the fitted circles are shifted with respect to the origin. For the higher modes, this shift is large compared to the radius of the circle. Therefore, the modulus of $\overline{\tilde{u}}_{z z}^{\mathrm{GS}}\left(k_{r}, \omega\right)$ is affected significantly. As a result, the Rayleigh peak corresponding to the third mode is located between $\operatorname{Re}\left(k_{\mathrm{R} 3}^{\mathrm{T}}(\omega)\right)$ and $\operatorname{Re}\left(k_{\mathrm{R} 4}^{\mathrm{T}}(\omega)\right)$, as can be seen in figure $3 \mathrm{~b}$. There is no Rayleigh peak that corresponds to the fourth mode at $50 \mathrm{~Hz}$.

Comparing figures $5 \mathrm{~b}$ and figure $5 \mathrm{a}$, it is noticed that the discretization and truncation of the wavenumber transformation (20) only has a small influence on the angular sweep of the central angle of the Rayleigh circles. The estimates $k_{\mathrm{R} j}^{\mathrm{Sc}}(\omega)$ obtained with the circle fit method are therefore less influenced by this transformation than the estimates $k_{\mathrm{R} j}^{\mathrm{Sp}}(\omega)$ obtained with the peak picking 
method. As a result, $k_{\mathrm{R} 1}^{\mathrm{Sc}}(\omega)$ and $k_{\mathrm{R} 2}^{\mathrm{Sc}}(\omega)$ perfectly match $\operatorname{Re}\left(k_{\mathrm{R} 1}^{\mathrm{T}}(\omega)\right)$ and $\operatorname{Re}\left(k_{\mathrm{R} 2}^{\mathrm{T}}(\omega)\right)$. The difference between $k_{\mathrm{R} 3}^{\mathrm{Sc}}(\omega)$ and $\operatorname{Re}\left(k_{\mathrm{R} 3}^{\mathrm{T}}(\omega)\right)$ is noticeable, but $k_{\mathrm{R} 3}^{\mathrm{Sc}}(\omega)$ still remains more accurate than $k_{\mathrm{R} 3}^{\mathrm{Sp}}(\omega)$.

Figure 7a compares the estimates $C_{\mathrm{R} j}^{\mathrm{Sp}}(\omega)$ of the dispersion curves obtained by applying the peak picking method to the simulated fk-spectrum, with the theoretical dispersion curves $C_{\mathrm{R} j}^{\mathrm{T}}(\omega)$ and the curves $C_{\mathrm{R} j}^{\mathrm{Tp}}(\omega)$ obtained by applying the peak picking method to the analytical fk-spectrum, in the frequency range where $C_{\mathrm{R} j}^{\mathrm{Tp}}(\omega)$ were accurately identified. Figure $7 \mathrm{~b}$ similarly compares the estimates $C_{\mathrm{R} j}^{\mathrm{Sc}}(\omega)$ of the dispersion curves obtained by applying the circle fit method to the simulated fk-spectrum, with the theoretical dispersion curves $C_{\mathrm{R} j}^{\mathrm{T}}(\omega)$ and the curves $C_{\mathrm{R} j}^{\mathrm{Tc}}(\omega)$ obtained by applying the circle fit method to the analytical fk-spectrum, in the frequency range where $C_{\mathrm{R} j}^{\mathrm{Tc}}(\omega)$ was accurately identified. Both figures show that the dispersion curves of the first mode obtained with the peak picking method and the circle fit method both match $C_{\mathrm{R} 1}^{\mathrm{T}}(\omega)$ for frequencies above $10 \mathrm{~Hz}$. At frequencies below $10 \mathrm{~Hz}$, leakage makes it impossible to trace the dispersion curve. For the second mode, both $C_{\mathrm{R} 2}^{\mathrm{Sp}}(\omega)$ and $C_{\mathrm{R} 2}^{\mathrm{Sc}}(\omega)$ match $C_{\mathrm{R} 2}^{\mathrm{T}}(\omega)$ in the considered frequency range, but the circle fit method is more accurate. The same holds for the fourth Rayleigh mode. With the circle fit method, an almost perfect match is found between $C_{\mathrm{R} j}^{\mathrm{Sc}}(\omega)$ and $C_{\mathrm{R} j}^{\mathrm{Tc}}(\omega)$ for all modes at all identified frequencies above $10 \mathrm{~Hz}$. Although it was possible to accurately identify the third Rayleigh mode by applying the peak picking method to the analytical Green's function $\tilde{u}_{z z}^{\mathrm{G}}\left(k_{r}, \omega\right)$ at frequencies above $34 \mathrm{~Hz}$, this is not possible by applying the peak picking method to the simulated Green's function $\tilde{u}_{z z}^{\mathrm{GS}}\left(k_{r}, \omega\right)$ at frequencies below $60 \mathrm{~Hz}$. The estimates of the Rayleigh phase velocity $C_{\mathrm{R} 3}^{\mathrm{Sc}}(\omega)$ by means of the circle fit method are accurate for frequencies above $50 \mathrm{~Hz}$.

These results show that the circle fit method allows to determine the dispersion curves of multiple modes in a larger frequency range and with higher accuracy than the peak picking method. It is possible to determine accurate dispersion curves for frequencies above the spatial aliasing limit. It was shown in this section that the dispersion curves obtained by applying the circle fit method to a simulated fk-spectrum correspond better to the theoretical dispersion curves obtained by applying the circle fit method to the analytical fk-spectrum than to the theoretical dispersion curves obtained from the solution of an eigenvalue problem. Therefore, it is beneficial to use the circle fit method for the determination of both the theoretical and the experimental dispersion curves in an inversion procedure where the misfit between these curves is minimized to determine the shear wave velocity profile of a site.

(a)

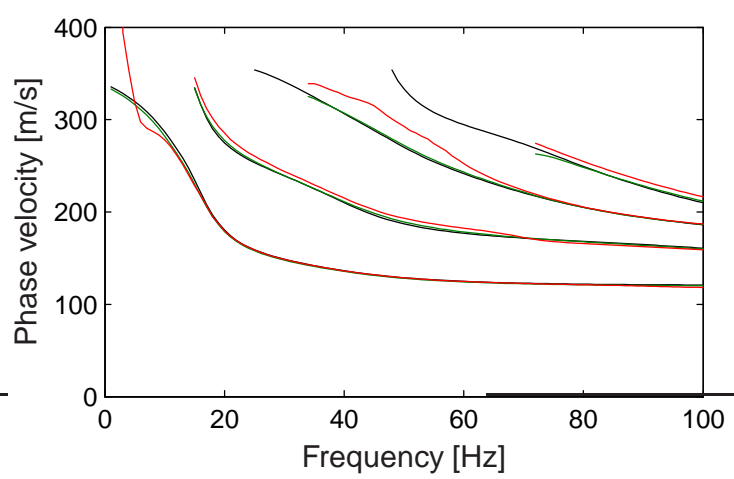

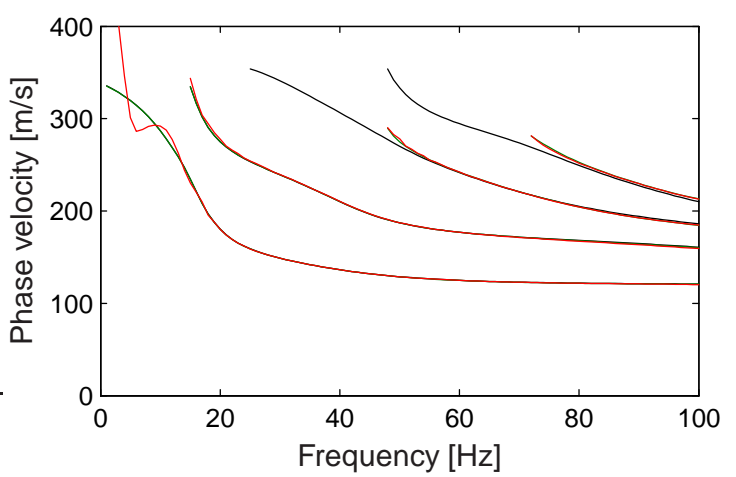

Figure 7. Theoretical Rayleigh dispersion curves $C_{\mathrm{R} j}^{\mathrm{T}}(\omega)(j=1,2,3,4)$ (black lines) and approximations (a) $C_{\mathrm{R} j}^{\mathrm{Tp}}(\omega)$ (green lines) and $C_{\mathrm{R} j}^{\mathrm{Sp}}(\omega)$ (red lines) obtained by applying the peak picking method and (b) $C_{\mathrm{R} j}^{\mathrm{Tc}}(\omega)$ (green lines) and $C_{\mathrm{R} j}^{\mathrm{Sc}}(\omega)$ (red lines) obtained by applying the circle fit method to the analytical and simulated fk-spectrum. 


\subsubsection{Rayleigh attenuation curves}

Comparison of figures $3 \mathrm{a}$ and $3 \mathrm{~b}$ shows that the wavenumber transformation results in a widening of the second Rayleigh peak and, consequently, an overestimation of the attenuation coefficient. This overestimation is visible at $50 \mathrm{~Hz}$ in figure $8 \mathrm{a}$, comparing $A_{\mathrm{R} j}^{\mathrm{Sh}}(\omega)$ obtained by applying the half-power bandwidth method to the simulated fk-spectrum, with $A_{\mathrm{R} j}^{\mathrm{T}}(\omega)$ obtained analytically, and $A_{\mathrm{R} j}^{\mathrm{Th}}(\omega)$ obtained by applying the half-power bandwidth method to the analytical fk-spectrum, in the same frequency range as the corresponding dispersion curves. A good correspondence between $A_{\mathrm{R} j}^{\mathrm{Sh}}(\omega)$ and $A_{\mathrm{R} j}^{\mathrm{T}}(\omega)$ or $A_{\mathrm{R} j}^{\mathrm{Th}}(\omega)$ is obtained only for the first mode, in the frequency range from 15 to $90 \mathrm{~Hz}$. The Rayleigh attenuation is overestimated at lower frequencies due to leakage and at higher frequencies due to aliasing. For the higher modes, no sufficient match is obtained between the estimated and theoretical curves.

Figure $8 \mathrm{~b}$ shows a similar comparison between $A_{\mathrm{R} j}^{\mathrm{Sc}}(\omega)$ obtained by applying the circle fit method to the simulated fk-spectrum, $A_{\mathrm{R} j}^{\mathrm{T}}(\omega)$, obtained analytically, and $A_{\mathrm{R} j}^{\mathrm{Tc}}(\omega)$, obtained by applying the circle fit method to the analytical fk-spectrum, in the same frequency range where the dispersion curves were obtained. For frequencies between 15 and $70 \mathrm{~Hz}$, there is a very good fit between $A_{\mathrm{R} j}^{\mathrm{Sc}}(\omega)$ and $A_{\mathrm{R} j}^{\mathrm{Tc}}(\omega)$ for all four modes, even if the correspondence between $A_{\mathrm{R} j}^{\mathrm{Tc}}(\omega)$ and $A_{\mathrm{R} j}^{\mathrm{T}}(\omega)$ is not good. For the first mode, there is a good fit for all considered frequencies above $15 \mathrm{~Hz}$. These results demonstrate that it is possible to use $A_{\mathrm{R} j}^{\mathrm{Tc}}(\omega)$ as an inversion target for a multi-modal inversion of Rayleigh attenuation curves and that the circle fit method is more robust than the half-power bandwidth method, which only results in a good fit for the first mode. The determination of attenuation curves is less accurate for frequencies above the spatial aliasing limit.

The circle fit method allows to determine the Rayleigh attenuation curves in a larger frequency range and for more modes than the half-power bandwidth method. For non-dominant modes, the estimates of the attenuation coefficients obtained with the circle fit method are more accurate than those obtained with the half-power bandwidth method. For the fundamental mode, the estimates are slightly less accurate. The circle fit method can also be used to determine the theoretical attenuation curves of a layered halfspace with known properties at a lower computational cost than required for the solution of the eigenvalue problem (4). Although these alternative attenuation curves do not fit the "true" attenuation curves that are solutions of this eigenvalue problem, they match very well with the attenuation curves estimated from a simulated wavefield experiment. Therefore, it is beneficial to use the circle fit method for the determination of both the theoretical
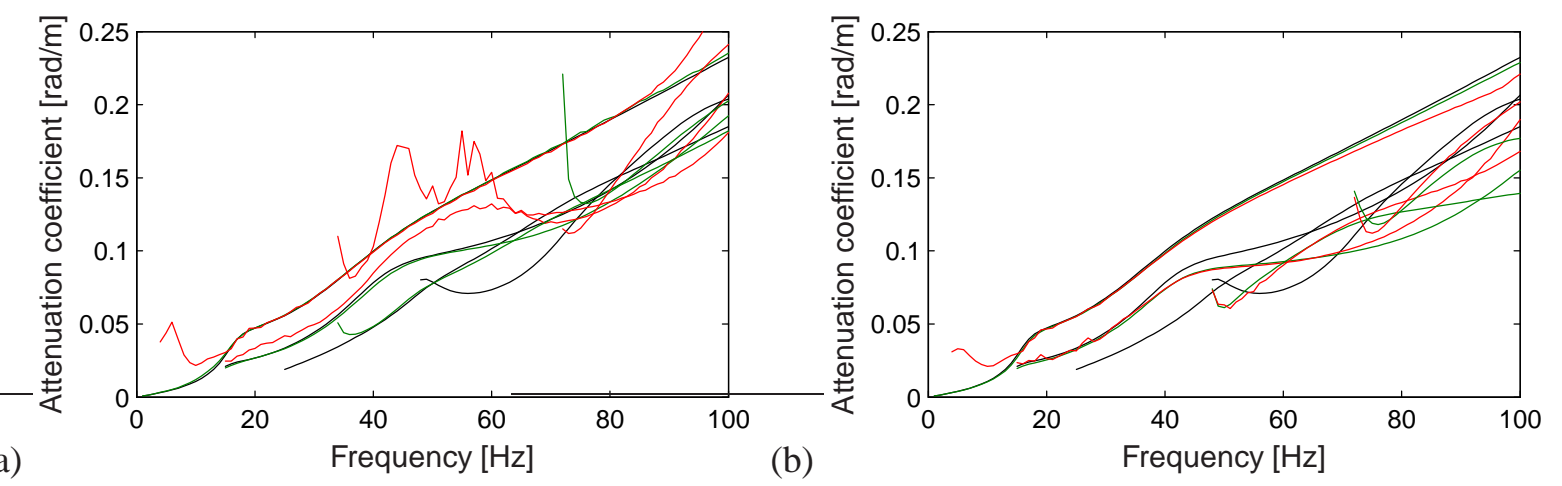

Figure 8. Theoretical Rayleigh attenuation curves $A_{\mathrm{R} j}^{\mathrm{T}}(\omega)(j=1,2,3,4)$ (black lines) and approximations (a) $A_{\mathrm{R} j}^{\mathrm{Th}}(\omega)$ (green lines) and $A_{\mathrm{R} j}^{\mathrm{Sh}}(\omega)$ (red lines) obtained by applying the half-power bandwidth method and (b) $A_{\mathrm{R} j}^{\mathrm{Tc}}(\omega)$ (green lines) and $A_{\mathrm{R} j}^{\mathrm{Sc}}(\omega)$ (red lines) obtained by applying the circle fit method to the analytical and simulated fk-spectrum. 
and the experimental attenuation curves in an inversion procedure were the misfit between these curves is minimized to determine the material damping ratio profile of a site. 


\subsection{Site with an irregular velocity profile}

\subsubsection{Simulated free field vibration}

The peaks in the analytical and simulated fk-spectrum of the Green's function of a regular soil profile with increasing shear wave velocity with depth usually correspond to well separated Rayleigh modes. In the case of an irregular soil profile where soft layers are trapped between stiffer layers, however, higher Rayleigh modes may significantly affect the fk-spectrum and the dominant Rayleigh wave (Gucunski \& Woods, 1992).

Figure 9a shows the normalized analytical Green's function $\overline{\tilde{u}}_{z z}^{\mathrm{G}}\left(k_{r}, \omega\right)$ and the dispersion curves $C_{\mathrm{R} j}^{\mathrm{T}}(\omega)$ of the first ten Rayleigh modes of the soil profile described in table 2, used in several benchmark studies on irregular soil profiles (Lai, 1998; Tokimatsu et al., 1992; Zomorodian \& Hunaidi, 2006); the shear wave velocity of the second layer is lower than in the first and third layer. As a result, the dominant Rayleigh peak in the fk-spectrum consists of multiple Rayleigh modes. At frequencies below $100 \mathrm{~Hz}$, the mode jumps can be clearly identified. At higher frequencies, however, it is not possible to distinguish different modes and the Rayleigh peak should be treated as an apparent or effective Rayleigh mode (Lai, 1998), which is affected by mode interaction. Figure $9 \mathrm{~b}$ shows the fk-spectrum of the normalized simulated Green's function $\overline{\tilde{u}}_{z z}^{\mathrm{GS}}\left(k_{r}, \omega\right)$, computed for a receiver setup with $\Delta r=1 \mathrm{~m}$ and $r_{\max }=80 \mathrm{~m}$, as well as the spatial aliasing limit. This figure clearly shows that it is possible to identify the Rayleigh dispersion curve at frequencies above the spatial aliasing limit.

\subsubsection{Rayleigh dispersion curves}

Figure 10a compares the estimate $C_{\mathrm{R} 1}^{\mathrm{Sp}}(\omega)$ of the first effective dispersion curve, obtained by applying the peak picking method to the simulated fk-spectrum, with the theoretical dispersion curve $C_{\mathrm{R} 1}^{\mathrm{Tp}}(\omega)$ obtained by applying the peak picking method to the analytical fk-spectrum. Figure $10 \mathrm{~b}$ similarly compares the estimate $C_{\mathrm{R} 1}^{\mathrm{Sc}}(\omega)$ of the dominant effective dispersion curve, obtained by applying the circle fit method to the simulated fk-spectrum, with the theoretical dispersion curve $C_{\mathrm{R} 1}^{\mathrm{Tc}}(\omega)$ obtained by applying the circle fit method to the analytical fk-spectrum. Around $5 \mathrm{~Hz}$, the Rayleigh dispersion curve descends steeply due to the large velocity contrast between the third layer and the underlying halfspace. Both figures show a very good match between the theoretical and simulated curve.

From the simulated fk-spectrum of the normalized analytical Green's function $\overline{\tilde{u}}_{z z}^{\mathrm{G}}\left(k_{r}, \omega\right)$ in figure 9a, other higher order effective Rayleigh modes can be determined from different Rayleigh peaks. Figure 11a compares the estimate $C_{\mathrm{R} 6}^{\mathrm{Sp}}(\omega)$ of the sixth effective dispersion curve, obtained by applying the peak picking method to the simulated fk-spectrum, with the theoretical dispersion
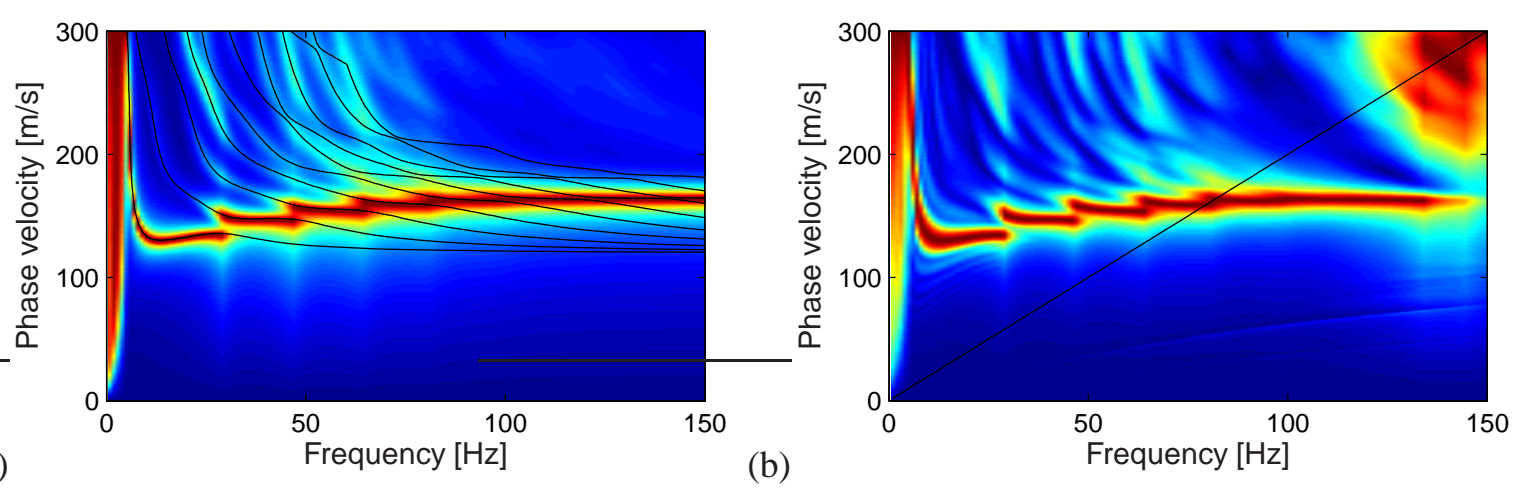

Figure 9. Fk-spectrum of (a) the normalized analytical Green's function $\overline{\tilde{u}}_{z z}^{\mathrm{G}}\left(k_{r}, \omega\right)$ with Rayleigh dispersion curves $C_{\mathrm{R} j}^{\mathrm{T}}(\omega)(j=1, \ldots, 10)$ (black lines) and (b) the normalized simulated Green's function $\overline{\tilde{u}}_{z z}^{\mathrm{GS}}\left(k_{r}, \omega\right)$ with spatial aliasing limit (black line), for the irregular soil profile. 
Table 2. Dynamic soil characteristics of the irregular soil profile.

\begin{tabular}{cccccc}
\hline Layer & $\begin{array}{c}d \\
{[\mathrm{~m}]}\end{array}$ & $\begin{array}{c}C_{\mathrm{s}} \\
{[\mathrm{m} / \mathrm{s}]}\end{array}$ & $\begin{array}{c}C_{\mathrm{p}} \\
{[\mathrm{m} / \mathrm{s}]}\end{array}$ & $\begin{array}{c}\beta_{\mathrm{s}}=\beta_{\mathrm{p}} \\
{[-]}\end{array}$ & $\begin{array}{c}\rho \\
{\left[\mathrm{kg} / \mathrm{m}^{3}\right]}\end{array}$ \\
\hline 1 & 2.0 & 180 & 300 & 0.03 & 1800 \\
2 & 4.0 & 120 & 200 & 0.03 & 1800 \\
3 & 8.0 & 180 & 1286 & 0.03 & 1800 \\
4 & $\infty$ & 700 & 1323 & 0.03 & 1800 \\
\hline
\end{tabular}

curve $C_{\mathrm{R} 6}^{\mathrm{Tp}}(\omega)$, obtained by applying the peak picking method to the analytical fk-spectrum. This apparent Rayleigh mode consists of 2 Rayleigh modes with a small mode jump from the sixth to the fifth mode at $63 \mathrm{~Hz}$. The fit between the two curves is slightly less good than was the case for the first effective dispersion curve. Figure $11 \mathrm{~b}$ similarly compares the estimate $C_{\mathrm{R} 6}^{\mathrm{Sc}}(\omega)$ of the sixth effective dispersion curve, obtained by applying the circle fit method to the simulated fkspectrum, with the theoretical dispersion curve $C_{\mathrm{R} 6}^{\mathrm{Tc}}(\omega)$ obtained by applying the circle fit method to the analytical fk-spectrum. A perfect match between the theoretical and simulated dispersion curve is obtained.

\subsubsection{Rayleigh attenuation curves}

Figure 12a compares the estimate $A_{\mathrm{R} 1}^{\mathrm{Sh}}(\omega)$ of the first effective attenuation curve, obtained by applying the half-power bandwidth method to the simulated fk-spectrum, with the theoretical attenuation curve $A_{\mathrm{R} 1}^{\mathrm{Th}}(\omega)$, obtained by applying the half-power bandwidth method to the analytical fk-spectrum. Good agreement is obtained for frequencies between $10 \mathrm{~Hz}$ and $120 \mathrm{~Hz}$, except in the neighbourhood of the Rayleigh mode jumps. The interaction between two close Rayleigh peaks results in an increased effective Rayleigh attenuation coefficient at frequencies close to the mode jumps.

Figure $12 \mathrm{~b}$ shows a similar comparison between the estimate $A_{\mathrm{R} 1}^{\mathrm{Sc}}(\omega)$ of the first effective attenuation curve, obtained by applying the circle fit method to the simulated fk-spectrum, with the theoretical attenuation curve $A_{\mathrm{R} 1}^{\mathrm{Tc}}(\omega)$, obtained by applying the circle fit method to the analytical fk-spectrum. A slightly better match is obtained at frequencies between $10 \mathrm{~Hz}$ and $140 \mathrm{~Hz}$. A good match is also obtained at frequencies close to the mode jumps above $50 \mathrm{~Hz}$. Because the mode jumps result in an increased apparent Rayleigh attenuation coefficient, inversion of apparent

(a)

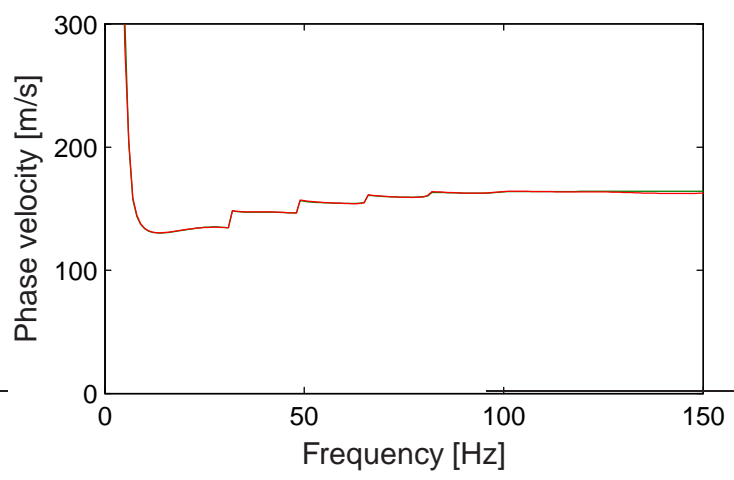

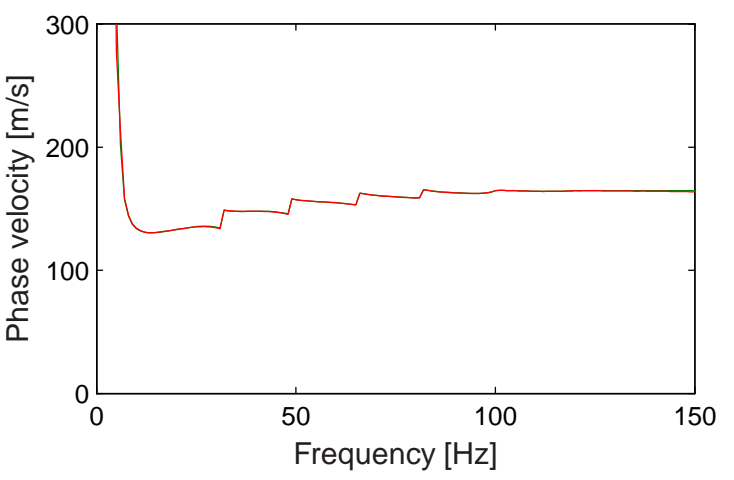

Figure 10. First effective Rayleigh dispersion curves (a) $C_{\mathrm{R} 1}^{\mathrm{Tp}}(\omega)$ (green line) and $C_{\mathrm{R} 1}^{\mathrm{Sp}}(\omega)$ (red line) obtained by applying the peak picking method and (b) $C_{\mathrm{R} 1}^{\mathrm{Tc}}(\omega)$ (green line) and $C_{\mathrm{R} 1}^{\mathrm{Sc}}(\omega)$ (red line) obtained by applying the circle fit method to the analytical and simulated fk-spectrum for the irregular soil profile. 

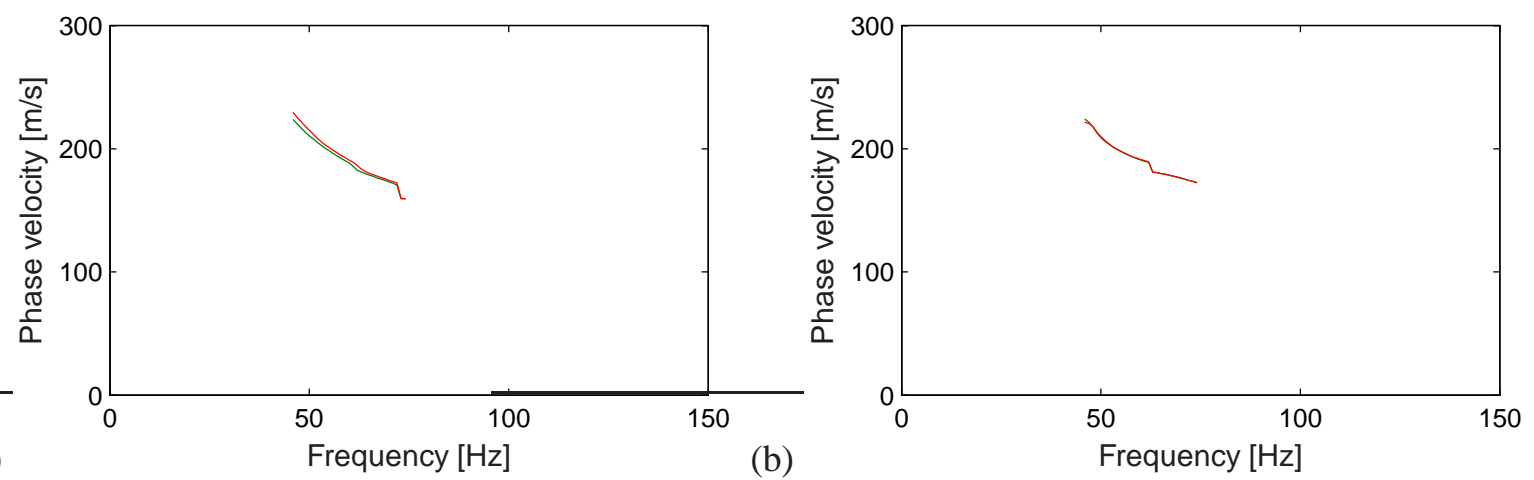

(a)

Figure 11. Sixth effective Rayleigh dispersion curve (a) $C_{\mathrm{R} 6}^{\mathrm{Tp}}(\omega)$ (green line) and $C_{\mathrm{R} 6}^{\mathrm{Sp}}(\omega)$ (red line) obtained by applying the peak picking method and (b) $C_{\mathrm{R} 6}^{\mathrm{Tc}}(\omega)$ (green line) and $C_{\mathrm{R} 6}^{\mathrm{Sc}}(\omega)$ (red line) obtained by applying the circle fit method to the analytical and simulated fk-spectrum for the irregular soil profile.

attenuation curves can help identifying the location of mode jumps. This information cannot be obtained from the Rayleigh dispersion curves.

Figure 13a compares the estimate $A_{\mathrm{R} 6}^{\mathrm{Sh}}(\omega)$ of the sixth effective attenuation curve, obtained by applying the half-power bandwidth method to the simulated fk-spectrum, with the theoretical attenuation curve $A_{\mathrm{R} 6}^{\mathrm{Th}}(\omega)$, obtained by applying the half-power bandwidth method to the analytical fk-spectrum. The agreement between the theoretical and simulated attenuation curve is not sufficient to be exploited in an inversion process.

Figure $13 \mathrm{~b}$ similarly compares the estimate $A_{\mathrm{R} 6}^{\mathrm{Sc}}(\omega)$ of the sixth effective attenuation curve, obtained by applying the circle fit method to the simulated fk-spectrum, with the theoretical attenuation curve $A_{\mathrm{R} 6}^{\mathrm{Tc}}(\omega)$, obtained by applying the circle fit method to the analytical fk-spectrum. The agreement between the effective Rayleigh attenuation coefficients obtained with the circle fit method is good. This example shows that the circle fit method, as opposed to the half-power bandwidth method, is also robust for the determination of the theoretical and experimental Rayleigh dispersion and attenuation curves in the case of an irregular soil profile.

\section{APPLICATION TO MEASUREMENT DATA}

\subsection{Experimental data}

All methods discussed in section 2 are applied to data collected at a site in Heverlee, Belgium. Based on geological information and CPT tests, it is concluded that the site consists of a quaternary

(a)

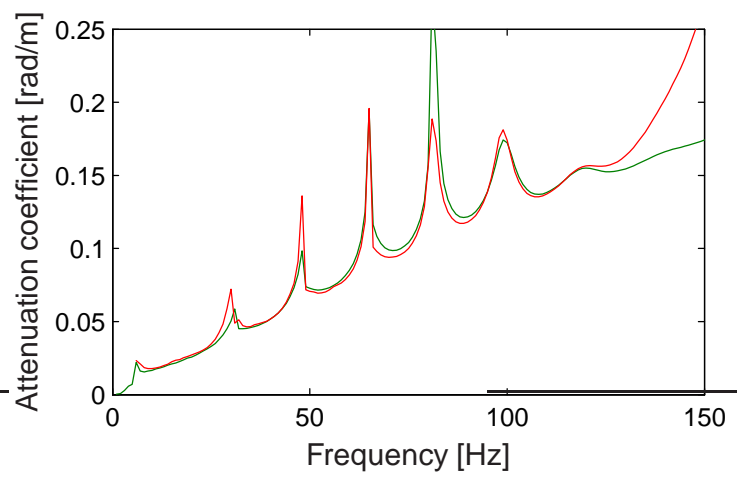

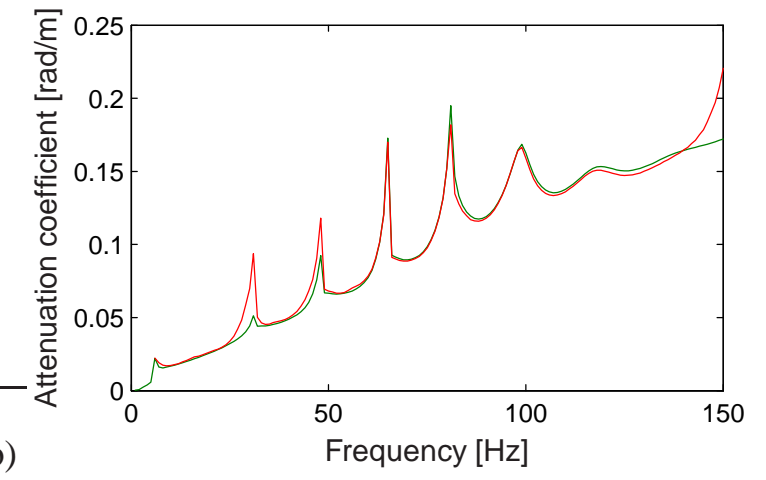

(b) (a) $A_{\mathrm{R} 1}^{\mathrm{Th}}(\omega)$ (green line) and $A_{\mathrm{R} 1}^{\mathrm{Sh}}(\omega)$ (red line) obtained by applying the half-power bandwidth method and (b) $A_{\mathrm{R} 1}^{\mathrm{Tc}}(\omega)$ (green line) and $A_{\mathrm{R} 1}^{\mathrm{Sc}}(\omega)$ (red line)
obtained by applying the circle fit method to the analytical and simulated fk-spectrum for the irregular soil profile. 

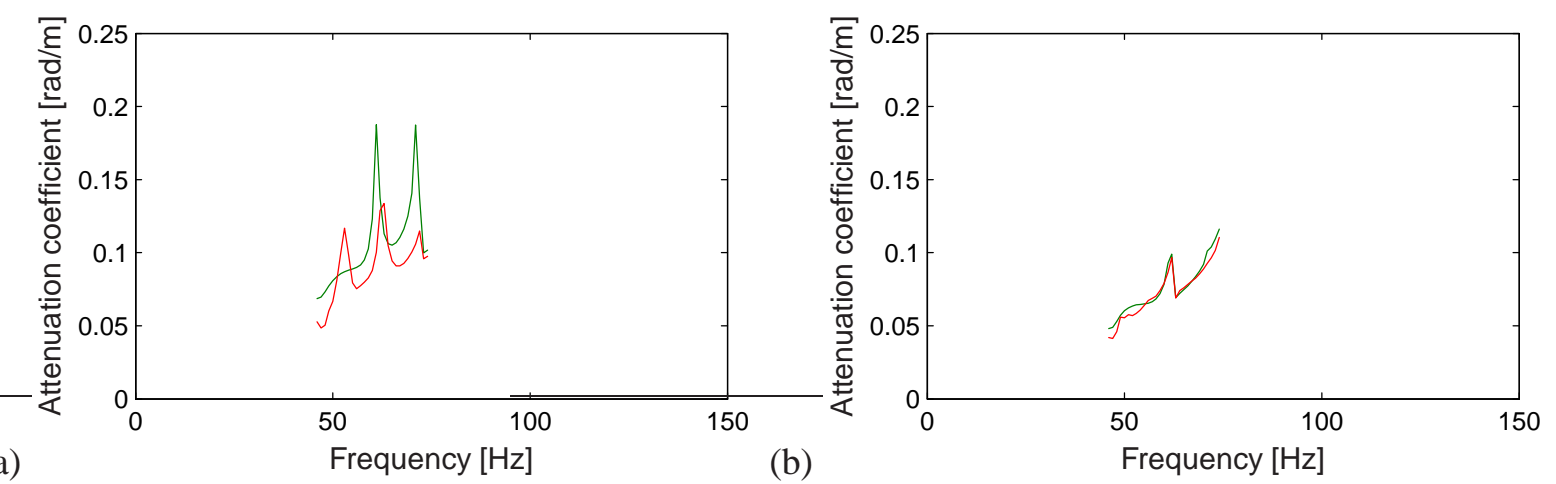

Figure 13. Sixth effective Rayleigh attenuation curves (a) $A_{\mathrm{R} 6}^{\mathrm{Th}}(\omega)$ (green line) and $A_{\mathrm{R} 6}^{\mathrm{Sh}}(\omega)$ (red line) obtained by applying the half-power bandwidth method and (b) $A_{\mathrm{R} 6}^{\mathrm{Tc}}(\omega)$ (green line) and $A_{\mathrm{R} 6}^{\mathrm{Sc}}(\omega)$ (red line) obtained by applying the circle fit method to the analytical and simulated fk-spectrum for the irregular soil profile.

layer with a thickness between $5 \mathrm{~m}$ and $15 \mathrm{~m}$ consisting of loose to densely packed sand, followed by a tertiary layer, consisting of medium dense to dense sand with sand stone concretions in the upper meters.

A MASW test was performed in September 2016 (Verachtert \& Degrande, 2017). Surface waves were generated by means of more than 100 hammer impacts on a $0.4 \mathrm{~m} \times 0.4 \mathrm{~m} \times 0.08 \mathrm{~m}$ aluminum foundation. The acceleration at the soil's surface was measured at 79 receiver positions between $1 \mathrm{~m}$ and $80 \mathrm{~m}$. The force-velocity transfer function $\hat{H}_{z z}^{\mathrm{E}}(r, \omega)$ is shown in figure $14 \mathrm{a}$. It is multiplied with the frequency spectrum of a hammer impact force, followed by an inverse Fourier transformation from the circular frequency $\omega$ to the time $t$, to obtain the free field accelerations $a_{z z}^{\mathrm{E}}(r, t)$ (figure 14b). The seismic traces are scaled individually, so that the effect of geometrical and material damping cannot be observed. The coherence between successive receivers and the signal to noise ratio are very good. The Rayleigh wave and its dispersive behaviour can clearly be observed. The dominant Rayleigh wave has an average velocity of $185 \mathrm{~m} / \mathrm{s}$. These time signals are used to determine the normalized experimental fk-spectrum $\overline{\tilde{H}}_{z z}^{\mathrm{E}}\left(k_{r}, \omega\right)$ (figure 14c), following the procedure explained in subsection 3.1.1. The fk-spectrum shows a clear peak with constant phase velocity for frequencies up to $78 \mathrm{~Hz}$, indicating that the surface response in this frequency range is dominated by the fundamental Rayleigh mode and that the site can be represented as a homogeneous halfspace. At frequencies below $5 \mathrm{~Hz}$, the accuracy of the fk-spectrum $\overline{\tilde{H}}_{z z}^{\mathrm{E}}\left(k_{r}, \omega\right)$ is too low for a reliable estimation of the dispersion curve $C_{\mathrm{R}}^{\mathrm{E}}(\omega)$. Consequently, the largest Rayleigh wavelength $\lambda_{\mathrm{Rmax}}^{\mathrm{E}}$ that can be measured is about $40 \mathrm{~m}$. At frequencies above $78 \mathrm{~Hz}$, the fk-spectrum is influence by spatial aliasing; the spatial aliasing wavelength equals $\lambda_{\mathrm{Rmin}}^{\mathrm{E}}=2 \Delta r=2 \mathrm{~m}$. Higher modes affected by spatial aliasing can be observed in figure 14c). Since the phase velocity of the

(a)

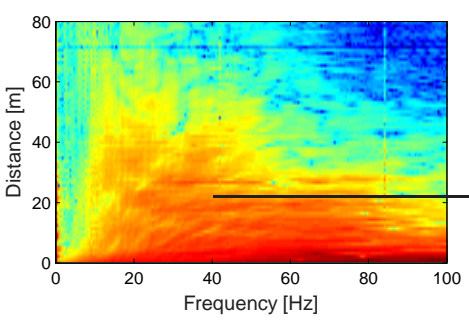

(b)

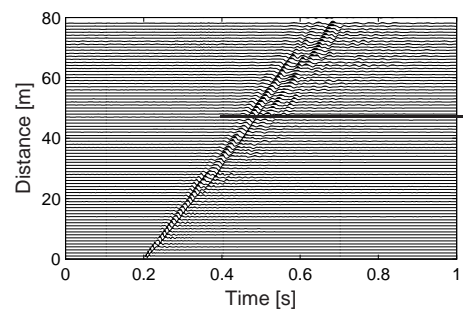

(c)

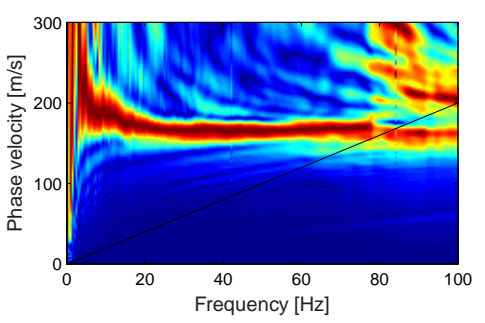

Figure 14. (a) Modulus of the force-velocity transfer function $\hat{H}_{z z}^{\mathrm{E}}(r, \omega)$, (b) acceleration $a_{z z}^{\mathrm{E}}(r, t)$ and (c) normalized experimental fk-spectrum $\overline{\tilde{H}}_{z z}^{\mathrm{E}}\left(k_{r}, \omega\right)$ and spatial aliasing limit (black line) for the site in Heverlee. 
(a)

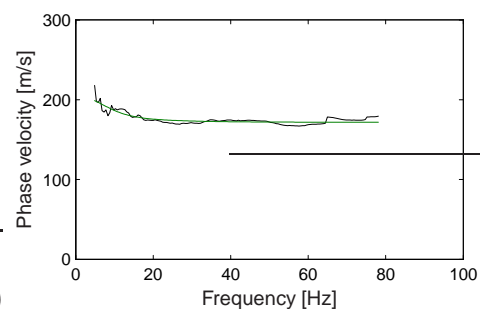

(b)

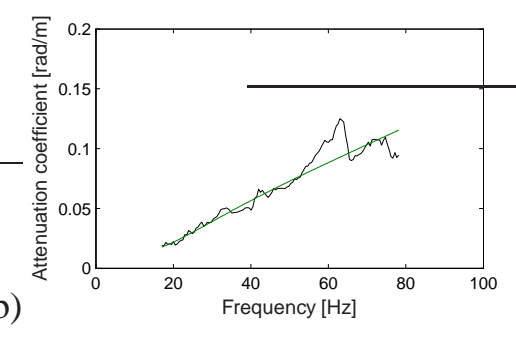

(c)

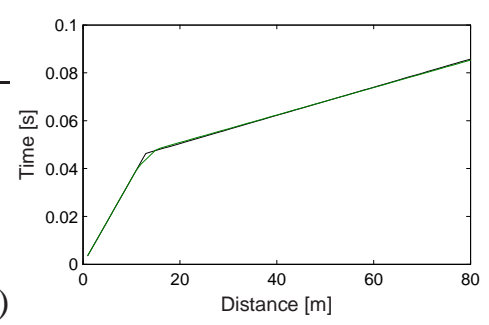

Figure 15. Experimental (black line) (a) dispersion curve, (b) attenuation curve and (c) first arrival time compared with the theoretical curves (green line) corresponding to soil profile 2. The experimental and theoretical dispersion and attenuation curves are determined by means of the circle fit method.

fundamental Rayleigh wave is predominantly determined by the properties of the soil at depths smaller than one third to one half of the Rayleigh wavelength, only the top $1 \mathrm{~m}$ of the soil has an influence on the dispersion curve. It is, however, likely that the soil near the surface is heterogeneous and local variations may have a significant influence on the high frequency response. Higher frequencies are, therefore, not taken into account.

The experimental dispersion curve $C_{\mathrm{R}}^{\mathrm{E}}(\omega)$ and attenuation curve $A_{\mathrm{R}}^{\mathrm{E}}(\omega)$ are determined by means of the methods discussed in section 2 . Figures $15 \mathrm{a}$ and $16 \mathrm{a}$ show the experimental dispersion curves $C_{\mathrm{R}}^{\mathrm{Ec}}(\omega)$ determined with the circle fit method and $C_{\mathrm{R}}^{\mathrm{Ep}}(\omega)$ determined with the peak picking method, respectively. $C_{\mathrm{R}}^{\mathrm{Ec}}(\omega)$ is slightly larger than $C_{\mathrm{R}}^{\mathrm{Ep}}(\omega)$, with a relative difference of less than $10 \%$. The theoretical curves of soil profiles 2 and 3 are determined in the next subsection as the solution of an inverse problem.

Figures $15 \mathrm{~b}$ and $16 \mathrm{~b}$ show the attenuation coefficient $A_{\mathrm{R}}^{\mathrm{Ec}}(\omega)$ determined with the circle fit method and $A_{\mathrm{R}}^{\mathrm{Eh}}(\omega)$ determined with the half-power bandwidth method, respectively. Due to leakage, the attenuation curves could only be identified for frequencies above $17 \mathrm{~Hz}$. $A_{\mathrm{R}}^{\text {Eh }}(\omega)$ is significantly larger than $A_{\mathrm{R}}^{\mathrm{Ec}}(\omega)$ in the whole frequency range.

It is not possible to determine which method is the most accurate by solely considering the experimental dispersion and attenuation curves. Both sets of curves are therefore used in an inversion procedure to determine the shear wave velocity and material damping ratio. The resulting soil profiles are used to simulate the force-velocity transfer functions. It will be shown in the following subsection that the soil profile obtained by inversion of the Rayleigh dispersion and attenuation curves determined by means of the circle fit method results in the most accurate force-velocity transfer functions.

In order to constrain the inverse problem, the first arrival times of the P-waves are also identified from the free field accelerations. A Short Term Averaging / Long Term Averaging (STA/LTA) procedure is followed in order to detect the first arrivals (Withers et al., 1998). The STA/LTA ratio

(a)

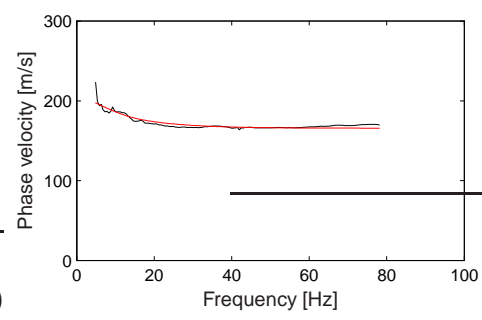

(b)

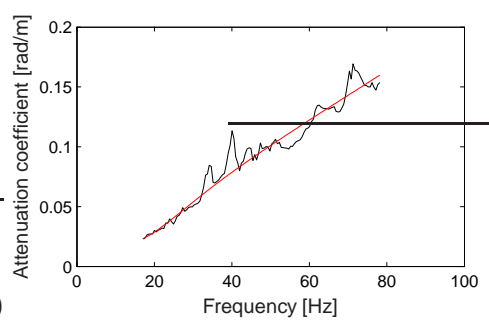

(c)

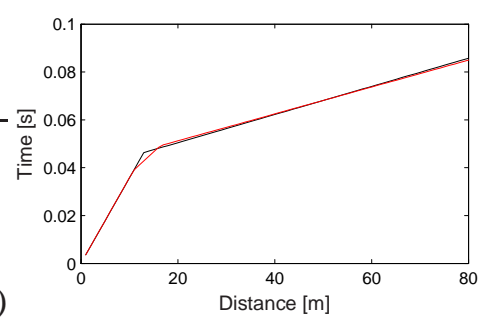

Figure 16. Experimental (black line) (a) dispersion curve, (b) attenuation curve and (c) first arrival time compared with the theoretical curves (red line) corresponding to soil profile 3 . The experimental and theoretical dispersion and attenuation curves are determined by means of the peak picking and half-power bandwidth method, respectively. 


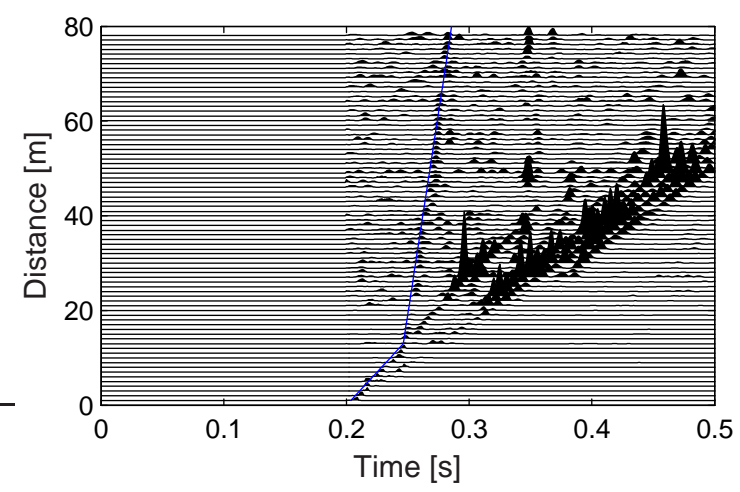

Figure 17. STA/LTA ratio $R(r, t)$ of the free field acceleration $a_{z z}^{\mathrm{E}}(r, t)$ and first arrival of the $\mathrm{P}$-waves (blue line).

$R(r, t)$ shown in figure 17 is computed as follows:

$R(r, t)=\frac{\frac{1}{N_{\mathrm{S}}} \sum_{k=0}^{N_{\mathrm{S}}-1} a_{z z}^{\mathrm{E}}{ }^{2}(r, t-k \Delta t)}{\frac{1}{N_{\mathrm{L}}} \sum_{k=N_{\mathrm{S}}}^{N_{\mathrm{S}}+N_{\mathrm{L}}-1} a_{z z}^{\mathrm{E}}{ }^{2}(r, t-k \Delta t)}$.

$N_{\mathrm{S}}=4$ and $N_{\mathrm{L}}=60$ are used in the present analysis. Only time samples after the pretrigger of $t=0.2 \mathrm{~s}$ are taken into account. The first arrival times are determined by fitting a bilinear curve through the local maxima which are assumed to correspond to the first arrival of the P-wave. The first arrival times are shown in figure 15c; this bilinear curve corresponds to a layered halfspace with a layer with a thickness of $5.5 \mathrm{~m}$ and a low P-wave velocity of $280 \mathrm{~m} / \mathrm{s}$ on top of a halfspace with a P-wave velocity of $1700 \mathrm{~m} / \mathrm{s}$.

\subsection{Inverse problem}

In order to determine the dynamic soil characteristics of the site, an inverse problem is solved. The design variables in the optimization scheme are the layer thickness $d$, the shear wave velocity $C_{\mathrm{s}}$, the ratio $C_{\mathrm{s}} / C_{\mathrm{p}}$ of the shear and dilatational wave velocity and the material damping ratio $\beta_{\mathrm{s}}$ (assumed to be identical for shear and dilatational waves). The density $\rho$ is kept fixed. The layer thickness $d$ is allowed to vary between $0.10 \mathrm{~m}$ and $10 \mathrm{~m}$, the shear wave velocity $C_{\mathrm{s}}$ between $50 \mathrm{~m} / \mathrm{s}$ and $1000 \mathrm{~m} / \mathrm{s}$, the ratio $C_{\mathrm{s}} / C_{\mathrm{p}}$ between 0.05 and 0.7 , and the material damping ratio $\beta_{\mathrm{s}}$ between 0.01 and 0.15 .

The objective function $f$ is formulated as follows:

$$
\begin{aligned}
f & =w_{\mathrm{C}} \sum_{k=1}^{N_{\mathrm{C}}}\left(\frac{C_{\mathrm{R}}^{\mathrm{T}}\left(\omega_{k}\right)-C_{\mathrm{R}}^{\mathrm{E}}\left(\omega_{k}\right)}{C_{\mathrm{R}}^{\mathrm{E}}\left(\omega_{k}\right)}\right)^{2}+w_{\mathrm{A}} \sum_{k=1}^{N_{\mathrm{A}}}\left(\frac{A_{\mathrm{R}}^{\mathrm{T}}\left(\omega_{k}\right)-A_{\mathrm{R}}^{\mathrm{E}}\left(\omega_{k}\right)}{A_{\mathrm{R}}^{\mathrm{E}}\left(\omega_{k}\right)}\right)^{2} \\
& +w_{\mathrm{T}} \sum_{k=1}^{N_{\mathrm{T}}}\left(\frac{T^{\mathrm{T}}\left(r_{k}\right)-T^{\mathrm{E}}\left(r_{k}\right)}{T^{\mathrm{E}}\left(r_{k}\right)}\right)^{2},
\end{aligned}
$$

where $C_{\mathrm{R}}^{\mathrm{T}}\left(\omega_{k}\right)$ and $A_{\mathrm{R}}^{\mathrm{T}}\left(\omega_{k}\right)$ are the theoretical surface wave velocity and attenuation coefficient at a frequency $\omega_{k}$ and $T^{\mathrm{T}}\left(r_{k}\right)$ is the theoretical first arrival time of the P-wave at distance $r_{k}$. The dispersion and attenuation curves are computed for $N_{\mathrm{C}}=151$ and $N_{\mathrm{A}}=126$ equidistant frequencies in the range shown in figures $15 \mathrm{a}$ and $15 \mathrm{~b}$, respectively. The first arrival times are computed at $N_{\mathrm{T}}=79$ receiver locations. Weighting factors $w_{\mathrm{C}}=w_{\mathrm{A}}=1$ and $w_{\mathrm{T}}=0.25$ are used.

This optimization problem is a non-linear least-squares problem, which is solved by means of the MATLAB function Isqnonlin (mat, 2011). This algorithm is a subspace trust-region method 
(a)

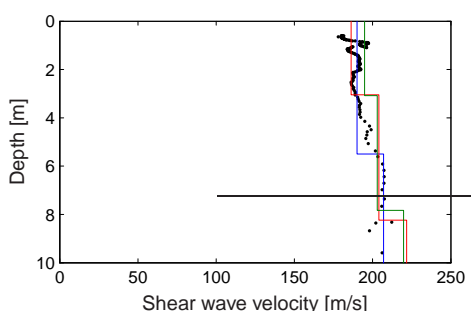

(b)

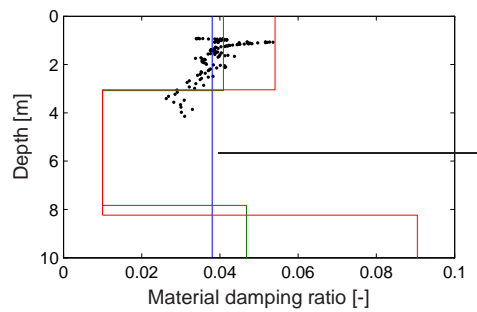

(c)

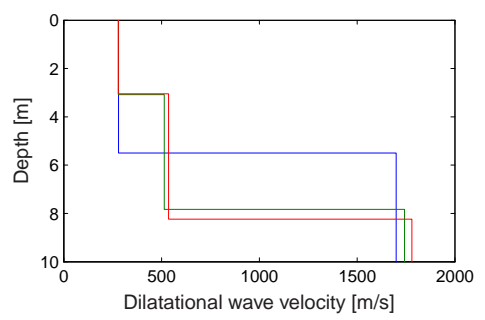

Figure 18. (a) Shear wave velocity, (b) material damping ratio and (c) dilatational wave velocity of soil profile 1 (blue line), 2 (green line) and 3 (red line). The black dots represent values obtained with rules of thumb.

and based on the interior-reflective Newton method. The sensitivities of the dispersion and attenuation curves with respect to the layer parameters are calculated analytically with the method described by Verachtert \& Degrande (2016).

First, an initial soil profile 1 is determined using rules of thumb (Foti et al., 2014). This profile is subsequently used as starting profile of an optimization process, based on experimental dispersion and attenuation curves determined with the circle fit method, in order to obtain soil profile 2. Finally, soil profile 3 is determined by solving an optimization problem based on the experimental dispersion and attenuation curves determined with the peak picking and half-power bandwidth method, respectively. The transfer functions computed for soil profiles 2 and 3 will finally be compared with the experimental transfer functions.

The shear wave velocity is estimated as $C_{\mathrm{s}}=1.1 C_{\mathrm{R}}^{\mathrm{Ec}}$ and the material damping ratio as $\beta_{\mathrm{s}}=$ $\lambda_{\mathrm{R}}^{\mathrm{Ec}} A_{\mathrm{R}}^{\mathrm{Ec}} / 2 \pi$, while the corresponding depth is estimated as $z=\lambda_{\mathrm{R}}^{\mathrm{Ec}} / 2.5$ with $\lambda_{\mathrm{R}}^{\mathrm{Ec}}=C_{\mathrm{R}}^{\mathrm{Ec}} / f$ the Rayleigh wavelength (Foti et al., 2014). The resulting shear wave velocity and material damping profile are indicated with dots on figures $18 \mathrm{a}$ and 18b, respectively. Based on these values, an initial soil profile 1 is proposed, consisting of two identical layers with a thickness of $2.75 \mathrm{~m}$, a shear wave velocity $C_{\mathrm{s}}=190 \mathrm{~m} / \mathrm{s}$ and a material damping ratio $\beta_{\mathrm{s}}=0.038$, on top of a halfspace with a shear wave velocity $C_{\mathrm{s}}=207 \mathrm{~m} / \mathrm{s}$ and a material damping ratio $\beta_{\mathrm{s}}=0.038$ (table 3 ). The total thickness of both layers equals the depth $5.5 \mathrm{~m}$ of the layer-halfspace interface in the P-wave velocity profile that results from the first arrival times of the P-waves (figure 18c).

During a first optimization, the misfit between the theoretical and experimental dispersion and attenuation curves determined with the circle fit method is minimized. The resulting soil profile 2 is shown on figure 18 and tabulated in table 3 . The shear wave velocity profile and material damping ratio of the first layer of soil profile 1 and 2 are very similar. The material damping ratio of the second layer of soil profile 2 is significantly reduced. As the attenuation curve could

Table 3. Dynamic soil characteristics of soil profiles 1, 2 and 3.

\begin{tabular}{ccccccc}
\hline Profile & Layer & $\begin{array}{c}\text { Thickness } \\
{[\mathrm{m}]}\end{array}$ & $\begin{array}{c}C_{\mathrm{s}} \\
{[\mathrm{m} / \mathrm{s}]}\end{array}$ & $\begin{array}{c}C_{\mathrm{p}} \\
{[\mathrm{m} / \mathrm{s}]}\end{array}$ & $\begin{array}{c}\beta_{\mathrm{s}}=\beta_{\mathrm{p}} \\
{[-]}\end{array}$ & $\begin{array}{c}\rho \\
{\left[\mathrm{kg} / \mathrm{m}^{3}\right]}\end{array}$ \\
\hline 1 & 1 & 2.75 & 190 & 280 & 0.038 & 1900 \\
& 2 & 2.75 & 190 & 280 & 0.038 & 1900 \\
& 3 & $\infty$ & 207 & 1700 & 0.038 & 1900 \\
\hline 2 & 1 & 3.1 & 195 & 280 & 0.041 & 1900 \\
& 2 & 4.7 & 203 & 514 & 0.010 & 1900 \\
& 3 & $\infty$ & 220 & 1743 & 0.047 & 1900 \\
\hline 3 & 1 & 3.0 & 186 & 280 & 0.054 & 1900 \\
& 2 & 5.2 & 204 & 543 & 0.010 & 1900 \\
& 3 & $\infty$ & 222 & 1773 & 0.091 & 1900 \\
\hline
\end{tabular}



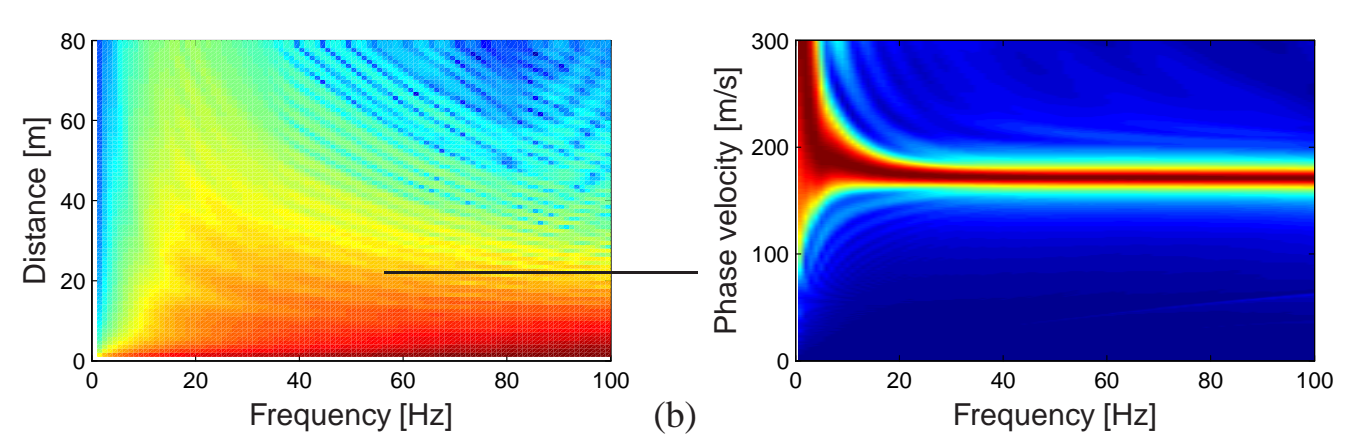

(a)

(b)

Figure 19. (a) Modulus of the simulated force-velocity transfer function $\hat{H}_{z z}(r, \omega)$ and (b) normalized simulated fk-spectrum $\overline{\tilde{H}}_{z z}\left(k_{r}, \omega\right)$ for soil profile 2 .

not be obtained at low frequencies, the material damping ratio of the halfspace is very uncertain. The dilatational wave velocity of the second layer is between the values of the first layer and the halfspace, which in turn remain both close to their initial values. The theoretical dispersion curve, attenuation curve and first arrival time corresponding to soil profile 2 show a very good fit with the experimental curves in figure 15. It should be noted that other soil profiles could also result in an acceptable fit between the theoretical and experimental curves. As the low frequency information is limited, the inverse problem is not sensitive to dynamic soil characteristics at larger depths; therefore, the depth and characteristics of the halfspace remain uncertain, especially the material damping ratio.

Soil profile 2 is used as a starting profile for a second inverse problem where the misfit between the theoretical and experimental dispersion and attenuation curves determined with the peak picking and half-power bandwidth method is minimized. The resulting soil profile 3 is shown on figure 18 and tabulated in table 3 . The main difference with soil profile 2 is the significantly higher material damping ratio in the first layer and the halfspace. The corresponding theoretical dispersion curve, attenuation curve and first arrival times are shown in figure 16. The correspondence between the theoretical and experimental dispersion curves and first arrival times is similar as for soil profile 2 . The fit between the experimental and theoretical attenuation curves of soil profile 3 is better above $50 \mathrm{~Hz}$, but less good below $50 \mathrm{~Hz}$.

Figure 19a shows that the simulated force-velocity transfer function $\hat{H}_{z z}(r, \omega)$ of soil profile 2 is similar to the experimental transfer function (figure 14a). Figure 19b shows the normalized simulated fk-spectrum $\overline{\tilde{H}}_{z z}\left(k_{r}, \omega\right)$ of soil profile 2 . The location and width of the peak in the simulated (figure 19b) and experimental (figure 14c) fk-spectrum correspond well. The higher modes appearing in the experimental fk-spectrum (figure 14c) are not present in the simulated fkspectrum. Figure 19a hence indicates that the identified soil profile 2 can reasonably well be used to predict vibrations below $80 \mathrm{~Hz}$. The modulus and fk-spectrum of the simulated force-velocity transfer function of soil profile 3 are very similar and therefore not shown.

In order to assess the correspondance between the experimental force-velocity transfer function $\hat{H}_{z z}^{\mathrm{E}}(r, \omega)$ and the simulated transfer functions $\hat{H}_{z z}^{\mathrm{S}}(r, \omega)$ for soil profile 2 and 3 in more detail, results are presented for four receiver locations in figure 20 . The $95 \%$ confidence region of the experimental transfer function is also indicated and shows that the measurement uncertainty is very large at frequencies below $15 \mathrm{~Hz}$. In the frequency range between $5 \mathrm{~Hz}$ and $80 \mathrm{~Hz}$, in the near field as well as in the far field, the simulated transfer function for soil profile 2 corresponds well with the experimental transfer function. At higher frequencies, the experimental and simulated transfer function also correspond reasonably well, although no information above $78 \mathrm{~Hz}$ was taken into account during the inversion procedure. Due to the increased material damping in soil 

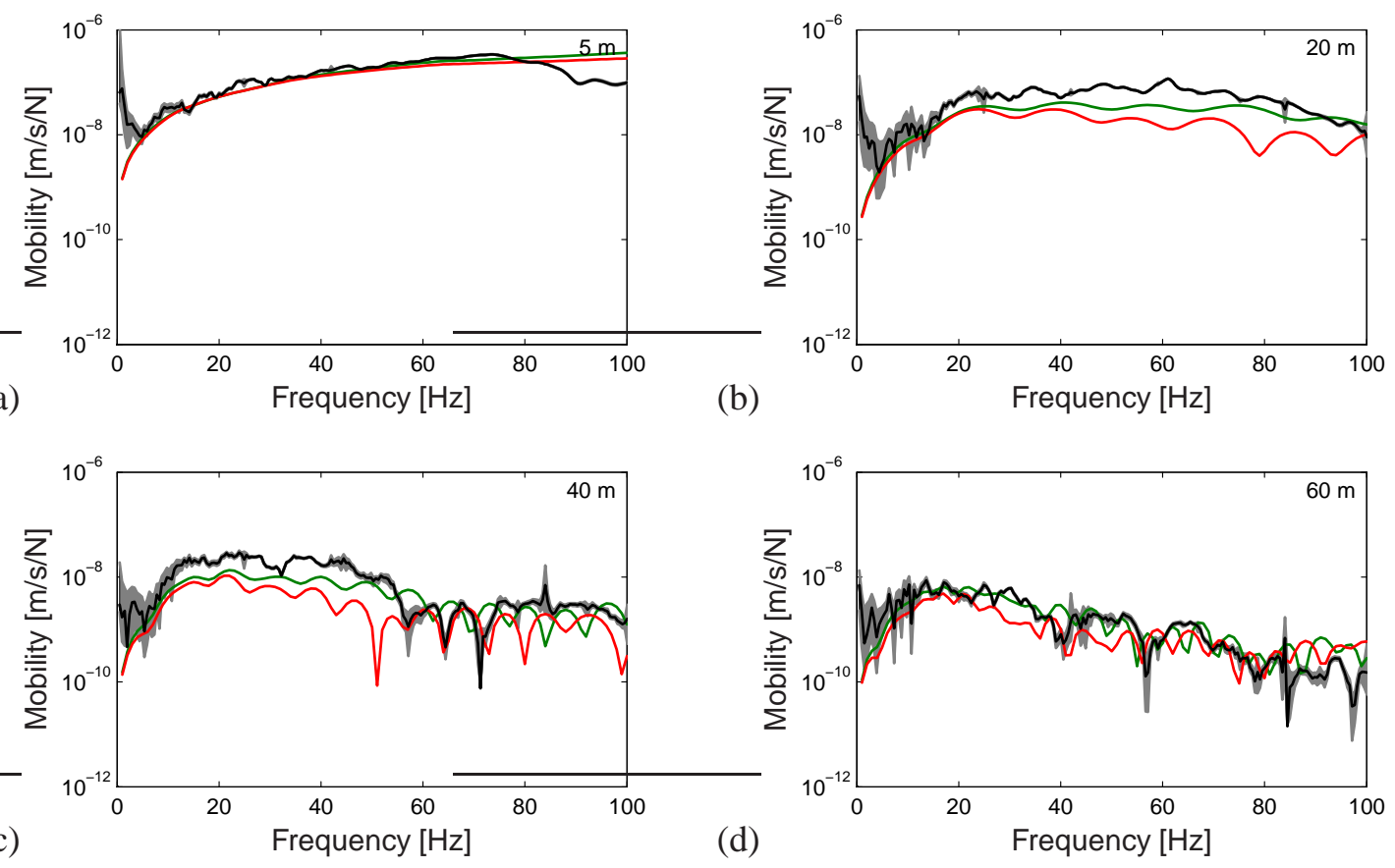

Figure 20. Modulus of the experimental force-velocity transfer function $\hat{H}_{z z}^{\mathrm{E}}(r, \omega)$ (black line) and simulated transfer function $\hat{H}_{z z}^{\mathrm{S}}(r, \omega)$ for soil profile 2 (green line) and 3 (red line) for a source-receiver distance of (a) $5 \mathrm{~m}$, (b) $20 \mathrm{~m}$, (c) $40 \mathrm{~m}$ and (d) $60 \mathrm{~m}$. The light grey area delimits the $95 \%$ confidence region of the experimental transfer function.

profile 3, the response is underestimated in the whole frequency range when compared to the experimental transfer function. In the frequency range below $5 \mathrm{~Hz}$, there is no agreement between the experimental and the simulated transfer functions, because the experimental force-velocity transfer functions are heavily affected by ambient noise. These results confirm that the circle fit method (used for the determination of soil profile 2) is more adequate for the determination and inversion of experimental dispersion and attenuation curves than the peak picking and half-power bandwidth methods (used for soil profile 3), because the attenuation curve determined with the half-power bandwidth method is overestimated in this experiment.

\section{CONCLUSION}

This paper introduces the circle fit method as a valuable method to determine both multi-modal experimental dispersion and attenuation curves as part of a MASW experiment. The proposed method employs the properties of the Nyquist plot of the Green's function in the fk-domain to determine the Rayleigh phase velocities and attenuation coefficients of multiple modes.

Two MASW experiments have been simulated in order to compare results obtained with the different methods for multi-modal determination of Rayleigh dispersion and attenuation curves. Both the peak picking and circle fit methods allow the determination of multi-modal (effective) dispersion curves, even at frequencies above the spatial aliasing limit. The simulated dispersion curves obtained with the circle fit method, however, are the most robust and agree better and in a wider frequency range with the theoretical dispersion curves, especially in the case of nondominant modes. Simulated (effective) attenuation curves determined with the half-power bandwidth method only agree with theoretical attenuation curves corresponding to dominant modes. Simulated (effective) attenuation curves determined with the circle fit method, on the other hand, agree well with theoretical attenuation curves of all identified modes. These results suggest that the 
circle fit method is more robust. The Rayleigh attenuation coefficients obtained with the circle fit method consequently are the most adequate target for a multi-modal inversion. Furthermore, it is observed that effective attenuation curves contain information on the mode jumps in the apparent Rayleigh dispersion curves.

Available methods as well as the proposed circle fit method are also applied to experimental data collected at a site in Heverlee, Belgium. The simulated force-velocity transfer function calculated for the soil profile based on the peak picking method and half-power bandwidth method underestimates the experimental transfer function, which indicates that the material damping ratio and Rayleigh attenuation coefficients are overestimated. The simulated force-velocity transfer function calculated for the soil profile based on the circle fit method shows a better agreement with the observed transfer functions, confirming that the circle fit method is more robust when applied on experimental data.

\section{ACKNOWLEDGMENTS}

The results presented in this paper have been obtained within the frame of the project OT/13/59 "Quantifying and reducing uncertainty in structural dynamics" funded by the Research Council of KU Leuven. The first author is a doctoral fellow of the Research Foundation Flanders (FWO). The financial support is gratefully acknowledged.

\section{REFERENCES}

2011. MATLAB Optimization Toolbox User's Guide, The MathWorks.

Aki, K. \& Richards, P., 2002. Quantitative seismology, University Science Books, Sausalito, California, 2nd edn.

Badsar, S., Schevenels, M., Haegeman, W., \& Degrande, G., 2010. Determination of the damping ratio in the soil from SASW tests using the half-power bandwidth method, Geophysical Journal International, 182(3), 1493-1508.

Ewins, D., 1984. Modal testing: theory and practice, Research Studies Press Ltd., Letchworth, UK.

Fladung, W. \& Rost, R., 1997. Application and correction of the exponential window for frequency response functions, Mechanical Systems and Signal Processing, 11(1), 23-36.

Forbriger, T., 2003. Inversion of shallow-seismic wavefields: I. Wavefield transformation, Geophysical Journal International, 153(3), 719-734.

Foti, S., 2004. Using transfer function for estimating dissipative properties of soils from surface-wave data, Near Surface Geophysics, 2(4), 231-240.

Foti, S., Parolai, S., Albarello, D., \& Picozzi, M., 2011. Application of surface-wave methods for seismic site characterization, Surveys in Geophysics, 32(6), 777-825.

Foti, S., Lai, C., Rix, G., \& Strobbia, C., 2014. Surface Wave methods for Near-Surface Site Characterization, CRC Press, Boca Raton, FL, USA.

Frazer, L. \& Gettrust, J., 1984. On a generalization of Filon's method and the computation of the oscillatory integrals of seismology, Geophysical Journal of the Royal Astronomical Society, 76, 461-481.

Gucunski, N. \& Woods, R., 1992. Numerical simulation of the SASW test, Soil Dynamics and Earthquake Engineering, 11, 213-227.

Karl, L., 2005. Dynamic soil properties out of SCPT and bender element tests with emphasis on material damping, Ph.D. thesis, Universiteit Gent.

Karl, L., Haegeman, W., \& Degrande, G., 2006. Determination of the material damping ratio and the shear wave velocity with the Seismic Cone Penetration Test, Soil Dynamics and Earthquake Engineering, 26(12), 1111-1126.

Kausel, E. \& Assimaki, D., 2002. Seismic simulation of inelastic soils via frequency-dependent moduli and damping, ASCE Journal of Engineering Mechanics, 128(1), 34-47.

Kausel, E. \& Roësset, J., 1981. Stiffness matrices for layered soils, Bulletin of the Seismological Society of America, 71(6), 1743-1761.

Lai, C., 1998. Simultaneous inversion of Rayleigh phase velocity and attenuation for near-surface site characterization, Ph.D. thesis, Georgia Institute of Technology.

Lai, C., Rix, G., Foti, S., \& Roma, V., 2002. Simultaneous measurement and inversion of surface wave dispersion and attenuation curves, Soil Dynamics and Earthquake Engineering, 22(9-12), 923-930. 
Misbah, A. \& Strobbia, C., 2014. Joint estimation of modal attenuation and velocity from multichannel surface wave data, Geophysics, 79(3), EN25-EN38.

Park, C., Miller, R., \& Xia, J., 1999. Multichannel analysis of surface waves, Geophysics, 64(3), 800-808.

Rizzo, F. \& Shippy, D., 1971. An application of the correspondence principle of linear viscoelasticity theory, SIAM Journal on Applied Mathematics, 21(2), 321-330.

Schevenels, M., 2007. The impact of uncertain dynamic soil characteristics on the prediction of ground vibrations, Ph.D. thesis, Department of Civil Engineering, KU Leuven.

Schevenels, M., Badsar, S., \& Degrande, G., 2008. Application of the SASW method for the determination of stiffness and damping parameters of soils, International Seminar on Interaction Soil-Railway-Track for High Speed Railways. Geotechnical aspects. LNEC, Lisbon, Portugal. Invited lecture.

Schevenels, M., François, S., \& Degrande, G., 2009. EDT: An ElastoDynamics Toolbox for MATLAB, Computers \& Geosciences, 35(8), 1752-1754.

Socco, L. \& Strobbia, C., 2004. Surface-wave method for near-surface characterization: a tutorial, Near Surface Geophysics, 2(4), 165-185.

Socco, L., Foti, S., \& Boiero, D., 2010. Surface-wave analysis for building near-surface velocity models: Established approaches and new perspectives, Geophysics, 75(5), 83-102.

Tokimatsu, K., Tamura, S. \& Kosjima, H., 1992. Effects of multiple modes on Rayleigh wave dispersion characteristics, Journal of Geotechnical Engineering, Proceedings of the ASCE, 118(10), 1529-1543.

Verachtert, R. \& Degrande, G., 2016. Computation of sensitivities of Rayleigh phase velocity and attenuation coefficient with the adjoint method, in 78th EAGE Conference \& Exhibition 2016, Vienna, Austria.

Verachtert, R. \& Degrande, G., 2017. Determination of the dynamic soil characteristics at site 1 next to the Blok D building of the administrative complex of KU Leuven, Report BWM-2017-03, Department of Civil Engineering, KU Leuven, Project OT/13/59.

Withers, M., Aster, R., Young, C., Beiriger, J., Harris, M., Moore, S., \& Trujillo, J., 1998. A comparison of select trigger algorithms for automated global seismic phase and event detection, Bulletin of the Seismological Society of America, 88(1), 95-106.

Xia, J., Miller, R., Park, C., \& Tian, G., 2002. Determining Q of near-surface materials from Rayleigh waves, Journal of Applied Geophysics, 51(2-4), 121-129.

Xia, J., Miller, R., Park, C., \& Tian, G., 2003. Inversion of high frequency surface waves with fundamental and higher modes, Journal of Applied Geophysics, 52(1), 45-57.

Zomorodian, S.M.A. \& Hunaidi, O., 2006. Inversion of SASW dispersion curves based on maximum flexibility coefficients in the wave number domain, Soil Dynamics and Earthquake Engineering, 26(1), 735-752.

Zywicki, D. \& Rix, G., 2005. Mitigation of near-field effects for seismic surface wave velocity estimation with cylindrical beamformers, Journal of Geotechnical and Geoenvironmental Engineering, Proceedings of the ASCE, 131(8), 970-977. 


\section{APPENDIX A: CIRCLE FIT ALGORITHM FOR THE DETERMINATION OF RAYLEIGH DISPERSION CURVES}

The algorithm is a local search algorithm with fixed step size, which finds the nearest local maximum close to an initial guess of the Rayleigh wavenumber. After the local maximum is found, the search step size is refined for better accuracy.

1. Choose an initial guess $k_{\mathrm{R} j}^{\mathrm{Tc} 0}(\omega)$ and a step size $\Delta k_{r}$.

2. Choose a number of steps $2 N$ for the search region.

3. Calculate the $L_{2}$-norm $L_{2}(n)=\left|\tilde{u}_{z z}^{\mathrm{G}}\left(k_{\mathrm{R} j}^{\mathrm{Tc} 0}(\omega)+n \Delta k_{r}, \omega\right)-\tilde{u}_{z z}^{\mathrm{G}}\left(k_{\mathrm{R} j}^{\mathrm{Tc} 0}(\omega)+(n-1) \Delta k_{r}, \omega\right)\right|$ for $n=-N+1,-N+2, \ldots, N$.

4. Determine $n_{\max }$ for which $L_{2}\left(n_{\max }\right)$ is maximum.

5. If $n_{\max }=-N+1$, then $k_{\mathrm{R} j}^{\mathrm{Tc} 0}(\omega)=\tilde{u}_{z z}^{\mathrm{G}}\left(k_{\mathrm{R} j}^{\mathrm{Tc} 0}(\omega)-N \Delta k_{r}, \omega\right)$ and return to step 3 . If $n_{\max }=N$, then $k_{\mathrm{R} j}^{\mathrm{Tc} 0}(\omega)=\tilde{u}_{z z}^{\mathrm{G}}\left(k_{\mathrm{R} j}^{\mathrm{Tc} 0}(\omega)+N \Delta k_{r}, \omega\right)$ and return to step 3 . Else, $k_{\mathrm{R} j}^{\mathrm{Tc} 0}(\omega)=k_{\mathrm{R} j}^{\mathrm{Tc} 0}(\omega)+\left(n_{\max }-1 / 2\right) \Delta k_{r}$.

6. If $\Delta k_{r}<\epsilon$, where $\epsilon$ is the desired wavenumber resolution, go step 7, else refine the step size $\Delta k_{r}$ and return to step 2.

7. Obtain the circle fit estimate of the Rayleigh wavenumber $k_{\mathrm{R} j}^{\mathrm{Tc}}(\omega)=k_{\mathrm{R} j}^{\mathrm{Tc} 0}(\omega)$ and the phase velocity $C_{\mathrm{R} j}^{\mathrm{Tc}}(\omega)=\omega / k_{\mathrm{R} j}^{\mathrm{Tc}}(\omega)$.

This algorithm is simple and efficient if a good initial estimate is chosen. In this paper, $k_{\mathrm{R} j}^{\mathrm{Tc} 0}(\omega)=$ $k_{\mathrm{Rj}}^{\mathrm{Tp}}(\omega)$, which is usually close to the circle fit estimate and therefore a good starting point. The initial step size $\Delta k_{r}$ is chosen so that $\omega / k_{\mathrm{R} j}^{\mathrm{Tc} 0}(\omega)-\omega /\left(k_{\mathrm{R} j}^{\mathrm{Tc} 0}(\omega)+\Delta k_{r}\right)=0.5 \mathrm{~m} / \mathrm{s} . N=10$ is kept fixed throughout the algorithm. The search step size is refined once with a factor 5 . 


\section{APPENDIX B: CIRCLE FIT ALGORITHM FOR THE DETERMINATION OF RAYLEIGH ATTENUATION CURVES}

1. Determine $k_{\mathrm{R} j}^{\mathrm{Tc}}(\omega)$ and $A_{\mathrm{R} j}^{\mathrm{Th}}(\omega)$.

2. Choose a number of wavenumbers $2 N$ for the circle fitting.

3. Determine the location of the centre $\left(x_{\mathrm{R} j}, y_{\mathrm{R} j}\right)$ of the circle to be determined as a linear least squares fit through the points $\tilde{u}_{z z}^{\mathrm{G}}\left(k_{\mathrm{R} j}^{\mathrm{Tc}}(\omega)+n A_{\mathrm{R} j}^{\mathrm{Th}}(\omega) / 2 N, \omega\right)$, for $n=-N,-N+1, \ldots, N$.

4. Use equation (18) to calculate a mean estimate of the attenuation coefficient as follows:

$$
A_{\mathrm{R} j}^{\mathrm{Tc}}=\frac{1}{N^{2}} \sum_{m=1}^{N} \sum_{n=1}^{N} \frac{\left(k_{\mathrm{R} j}^{\mathrm{Tc}}+m A_{\mathrm{R} j}^{\mathrm{Th}} / 2 N\right)^{2}-\left(k_{\mathrm{R} j}^{\mathrm{Tc}}-n A_{\mathrm{R} j}^{\mathrm{Th}} / 2 N\right)^{2}}{2 k_{\mathrm{R} j}^{\mathrm{Tc}}\left[\tan \left(\frac{\left|\theta_{j}\left(k_{\mathrm{R} j}^{\mathrm{Tc}}+n A_{\mathrm{R} j}^{\mathrm{Th}} / 2 N\right)\right|}{2}\right)+\tan \left(\frac{\left|\theta_{j}\left(k_{\mathrm{R} j}^{\mathrm{Tc}}(\omega)-n A_{\mathrm{R} j}^{\mathrm{Th}} / 2 N\right)\right|}{2}\right)\right]} .
$$

The range of wavenumbers selected to fit the circle corresponds to about $30 \%$ of a full circle and is chosen large enough to obtain a good circle fit, but not too large, in order to avoid interference with other Rayleigh wave modes. In this paper, $N=20$ is used. 